\title{
Nanocouplers for Infrared and Visible Light
}

\author{
A. Andryieuski and A. V. Lavrinenko \\ DTU Fotonik, Technical University of Denmark, Oersteds Plads 343, 2800 Kongens Lyngby, Denmark
}

Correspondence should be addressed to A. Andryieuski, andra@fotonik.dtu.dk

Received 14 June 2012; Accepted 6 September 2012

Academic Editor: Pavel A. Belov

Copyright () 2012 A. Andryieuski and A. V. Lavrinenko. This is an open access article distributed under the Creative Commons Attribution License, which permits unrestricted use, distribution, and reproduction in any medium, provided the original work is properly cited.

\begin{abstract}
An efficient and compact coupler-a device that matches a microwaveguide and a nanowaveguide-is an essential component for practical applications of nanophotonic systems. The number of coupling approaches has been rapidly increasing in the past ten years with the help of plasmonic structures and metamaterials. In this paper we overview recent as well as common solutions for nanocoupling. More specifically we consider the physical principles of operation of the devices based on a tapered waveguide section, a direct coupler, a lens, and a scatterer and support them with a number of examples.
\end{abstract}

\section{Introduction}

Photonic components have advantages comparing to the electronic ones. Infrared and optical frequencies $10^{14}$ $10^{15} \mathrm{~Hz}$ provide much broader operational bandwidth than the fastest electronic circuits. The losses in optical waveguides are smaller than in metallic wires. This is why, as D. Miller wrote, "the optical interconnects are progressively replacing wires" [1]. To achieve larger functionality on an integrated optical chip the optical components have to be miniaturized. A natural limitation, however, comes into play: the diffraction limit claims that we cannot focus light in a spot less than a half of the wavelength. The transverse size of conventional dielectric waveguides (e.g., silicon waveguides) is also limited to a half of the wavelength. Only employment of metals allows to overcome the diffraction limit and to confine a wave to a smaller area, very often at the cost of increased propagation losses.

Nevertheless, the problem is not only to create efficient waveguides that provide subwavelength mode confinement, but also to make an efficient interface between free space or an optical fiber and a subwavelength nanowaveguide, that is, to focus light and launch it efficiently into the waveguide. The artistic view of the situation is depicted in Figure 1. Trying to pour water from a big bowl into a bottle with a narrow bottleneck, one would waste a lot. However, usage of a funnel simplifies the task and increases the efficiency significantly. An optical coupler plays the role of a funnel for light.

The problem of optical coupling originates from the pronounced modal mismatch between an optical fiber (a conventional single-mode telecommunication fiber has the core of $8 \mu \mathrm{m}$ in diameter) and a nanosized waveguide, which has a core less than $1 \mu \mathrm{m}$. They have a small overlap of modal fields that prevents from the efficient coupling. Long adiabatically tapered fibers can solve the coupling problem of a subwavelength waveguide, but for an efficient mode conversion the tapered region may reach the length of several millimeters that is totally incompatible with modern microand nanofabrication. The compact efficient subwavelength couplers require new approaches.

We are witnessing a real explosion of various metamaterial and plasmonic solutions for focusing and nanocoupling in recent years. The reasons for that are first of all the extraordinary optical properties the artificial metal-dielectric structures offer. Not only the proposed device geometries are different, but also the very physical principles they are based on vary significantly.

The goal of this paper is to give an overview of existing nanocoupling solutions for the visible and near-infrared (telecom) range and to reveal the most efficient ones. We wish to focus mostly on the physical effects employed for nanocoupling rather than on technical details of the devices. 


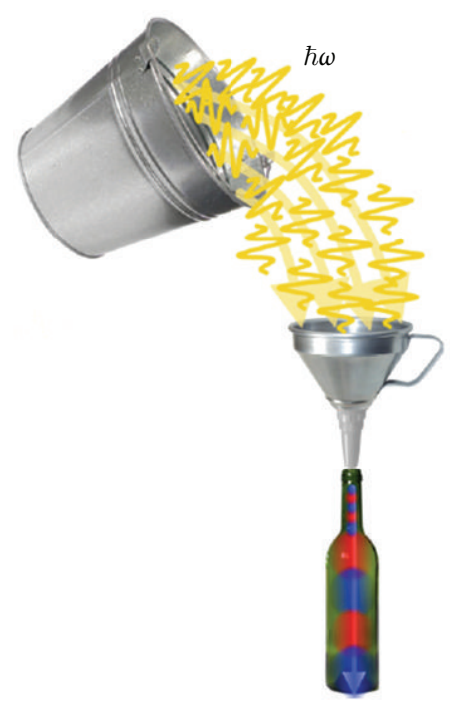

Figure 1: An artistic view of the problem of coupling light from a wide microscopic fiber to a nanoscopic waveguide. Employment of a coupler, which is represented by a funnel on the figure, minimizes the losses and simplifies optical alignment.

We are not going to provide the complete set of references on nanocouplers, since with heaps of articles already published and being published every month it is almost impossible. The cited papers should be considered rather as valuable examples of each physical effect employment.

The paper is organized as follows. In Section 2 the definitions of a nanocoupler and a nanowaveguide are given as well as the basic information on the common waveguide properties. Various physical principles, on which the nanocoupler realization can be grounded, are listed in Section 3. The tapered waveguide-based nanocouplers are discussed in Section 4. Section 5 deals with the directional nanocouplers. The lenses-based and scatterer-based couplers are considered in Section 6 and Section 7 correspondingly. Other ideas are listed in Section 8. Conclusions summarize the paper.

\section{Definitions}

2.1. Nanowaveguide. We define a nanowaveguide as an electromagnetic waveguide that has lateral sizes in the "nano" range, that is, $1-1000 \mathrm{~nm}$. Depending on the waveguide material and the wavelength of the light nanowaveguide can be subwavelength or not. The modal size of the subwavelength waveguide is smaller than the size of a focused light beam can be. The nanowaveguides can be divided in two groups: dielectric and plasmonic waveguides. Their combinations, the so-called hybrid waveguides, are also possible.

2.2. Dielectric Waveguide. The most common material for dielectric waveguides is silicon due to its high refractive index, transparency at the telecom wavelengths, and CMOS compatibility. Typically, silicon waveguides are rib, ridge, and photonic crystal waveguides. The high refractive index of the silicon waveguide makes it possible to reduce the crosssection down to $200 \times 500 \mathrm{~nm}^{2}$. The dielectric waveguide always has a cut-off size, below which the waveguide modes become leaky and the waveguide cannot transport light on reasonable distances.

Miniaturization of optoelectronic components requires decreasing the size of optical waveguides. However, the natural limitation comes into play. Due to diffraction the smallest size of an optical beam in a medium is on the order of the wavelength. If we consider a wave of the frequency $\omega$ propagating in the medium with the refractive index $n$, the wavenumber is $k=2 \pi n / \lambda$. The transverse wavevector components, for example, $k_{x}$ can take the values from $-k$ to $k$, so its maximal uncertainty can be $\Delta k_{x, \max }=$ $2 k=4 \pi n / \lambda$. Due to the uncertainty principle (or likewise Fourier transformations), the coordinate uncertainty $\Delta x$ is connected to the wavevector uncertainty $\Delta k_{x}$ [2] through

$$
\Delta x \Delta k_{x} \geq 2 \pi
$$

That limits the size of the light beam to

$$
\Delta x=\frac{2 \pi}{2 k}=\frac{\lambda}{2 n} \text {. }
$$

Considering, for example, the telecom wavelength $\lambda=$ $1.55 \mu \mathrm{m}$ and silica refractive index $n=1.5$ we can estimate the smallest size of a light beam as $\Delta x_{\min }=517 \mathrm{~nm}$. Light can be confined to smaller spots only by using surface plasmons at an interface between metal and dielectric.

2.3. Plasmonic Waveguide. Plasmonic waveguides are by default metal-dielectric waveguides. They attract a lot of attention since in some configurations they can show the absence of the cut-off wavelength at any waveguide size. Therefore, the mode size can be reduced to extremely small values but at the cost of increased optical losses. Another advantage of the plasmonic waveguides is the presence of metal that can be used not only as a waveguiding element but also as an electric contact that allows using it for tuning the dielectric surrounding (e.g., due to the electrooptical or thermooptical effects). Plasmonic waveguides are currently considered for the potential replacement of the electronic interconnects in the future generation integrated circuits.

Surface plasmon polaritons (SPPs) are the eigenmodes of a metal-dielectric interface. SPPs are combined lightelectrons density waves. On a flat metal (permittivity $\varepsilon_{1}$ )dielectric (permittivity $\varepsilon_{2}$ ) interface the SPPs are transverse waves with the magnetic field parallel to the interface. In the visible and near-infrared ranges the permittivity of metal is very dispersive. The propagating SPP solutions correspond to a case $\operatorname{Re}\left(\varepsilon_{1}+\varepsilon_{2}\right)<0$. For a detailed background on plasmonics theory and applications we refer the reader to $[3,4]$.

Several types of plasmonic waveguiding structures were proposed, for example, metal-insulator-metal and insulator-metal-insulator multilayered structures [5], strip [6], trenches and V-grooves $[7,8]$, wedge [9], slot $[10,11]$, and nanoparticles chain [12] waveguides. A comprehensive overview of the plasmonic waveguides and nanoplasmonic systems can be found in $[3,4,13-15]$. 


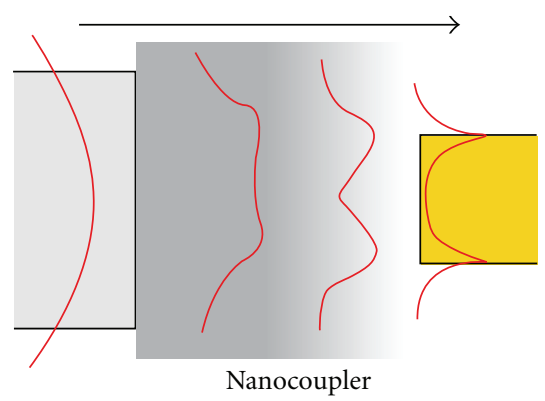

Figure 2: Nanocoupler concept: a focusing device or a mode convertor. As an example the mode of the dielectric waveguide (left) is gradually transformed into the mode of the plasmonic waveguide (right).

2.4. Nanocoupler Definition. A nanocoupler is a device that facilitates coupling of light from free space or a macroscopic optical fiber to a nanowaveguide. It can be understood as a focusing device, a sort of an optical funnel (see Figure 1) that squeezes light into a small spot. If we talk about matching a thick and a thin waveguides that have different modal distributions, the nanocoupler can be also understood as a mode convertor, a device that transforms a mode of the thick waveguide to a mode of the thin waveguide (see Figure 2).

2.5. Requirements to a Nanocoupler. We understand the nanocoupler as a focusing device or a mode convertor that gives a high coupling efficiency $(\mathrm{CE})$ which is the ratio of the power loaded into the waveguide $P_{\mathrm{WG}}$ to the power incident on the nanocoupler from free space or power delivered by a thick waveguide $P_{\text {inc }}$ :

$$
\mathrm{CE}=\frac{P_{\mathrm{WG}}}{P_{\mathrm{inc}}}
$$

High coupling efficiency automatically means low losses, including absorption $A$, reflection $R$, and scattering $S$. Additional requirements to the nanocoupler can be as follows.

(1) Small size on the order of several micrometers. This is an important requirement for the integration of the nanocoupler within an optical integrated circuit.

(2) Simplicity and small price of fabrication. This requirement is important for the mass production but not so important on the stage of the scientific development.

(3) Spectral selectivity. The importance of this property depends on the application. In many cases a broad bandwidth is desirable. However, there are some applications, where a narrow bandwidth is preferable, for example, if the coupler is used at the same time for the wavelength demultiplexing.

(4) Polarization sensitivity. The importance of this requirement depends on the application and on the selected nanowaveguide. If the nanowaveguide is polarization sensitive, the nanocoupler's working polarization should be matched with the polarization of the waveguide or it should be polarization insensitive.
We have to emphasize that the nanocoupler differs from a nanofocusing or a nanoimaging device. The goal of nanoimaging or nanofocusing is only to image a tiny light source or focus light into a small spot, respectively, no matter how large is the fraction of the transmitted power with respect to the incident power, while for the nanocoupler the coupling efficiency is the most crucial parameter. For example, the stimulated emission depletion technique $[16,17]$ allows for a fantastic resolution below $10 \mathrm{~nm}$ in the visible light imaging systems, but it can hardly be used for an efficient coupling.

\section{Nanocouplers Classification}

The role of the nanocoupler is to match the impedances (or wavevectors) and field profiles of an incident wave and a mode of the accepting nanowaveguide. Depending on the employed physical mechanisms several types of nanocouplers can be singled out.

(1) Tapered waveguides (Figure 3(a)) which will be referred to as tapered waveguide coupler. In this case a waveguide with the gradually reduced core crosssection compresses a wave towards the matching parameters with the accepting nanowaveguide.

(2) Light can be coupled first to a wide waveguide (e.g., a silicon or long-range surface plasmon-polariton waveguide) and then with the help of a directional coupler or a resonant stub coupled into a smaller nanowaveguide (Figure 3(b)). We will refer to this case as a direct coupler.

(3) Light can be tightly focused with a lens (Figure 3(c)). This is what we are referring to as a lens coupler.

(4) A single or multiple scatterers can be used for coupling (Figure 3(d)). Correspondingly, the device is called a scatterer coupler.

(5) There are some other ideas that do not fall into the previously mentioned categories. We will refer to them under a unified shield as other solutions.

The nanocoupler can be arranged as a separate device (Figure $4(\mathrm{a})$ ), as a device integrated with a waveguide on a chip (Figure 4(b)), or as a device integrated with an excitation fiber (Figure $4(\mathrm{c})$ ). For the practical applications the integrated configurations (b) and (c) are preferable, since a lesser number of movable parts simplifies optical alignment.

Two main excitation configurations are important in the practical applications: lateral coupling (Figure 5(a)), when the incident wave direction coincides with the nanowaveguide, and vertical coupling (Figure 5(b)), when the light direction is perpendicular to the nanowaveguide. The second configuration can provide not only incoupling from free space, but also communication of the optical elements between two layers of an optical integrated circuit. Incident angles of light different from 0 and 90 degrees are also possible. 


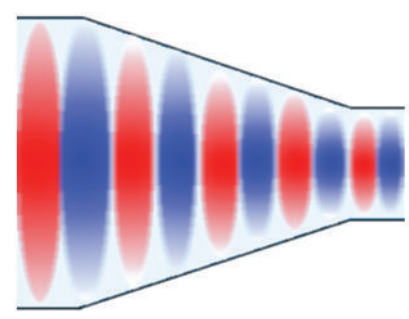

(a)

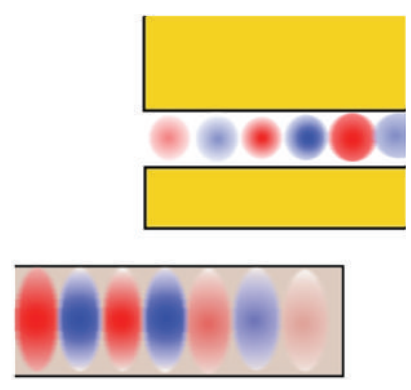

(b)

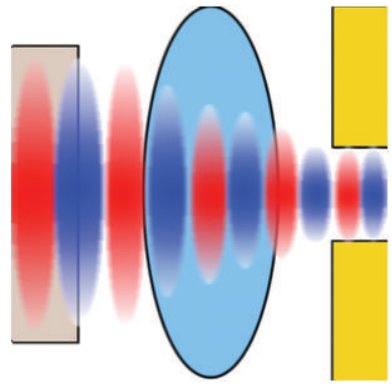

(c)

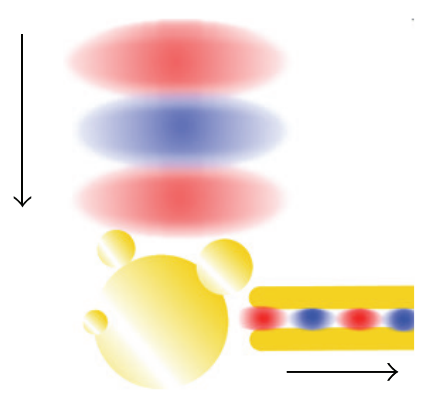

(d)

FIGURE 3: Types of the possible nanocouplers realizations: (a) tapered waveguide coupler, (b) direct coupler transferring the power from a wide waveguide to a narrow waveguide, (c) lens coupler, (d) scatterer coupler.

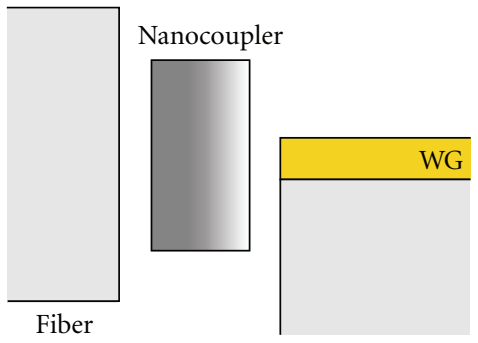

(a)
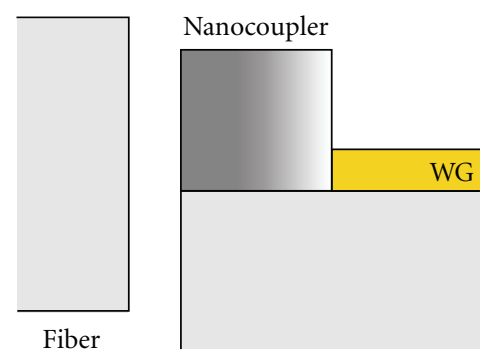

(b)

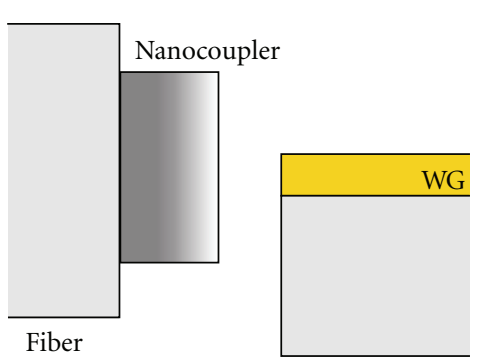

(c)

FIGURE 4: Possible geometrical configurations of the coupler: (a) a separate device, (b) integrated with a nanowaveguide (WG) on the same chip, (c) integrated with an excitation fiber.

\section{Tapered Waveguide Coupler}

As we already mentioned the tapered waveguide coupler is nothing else but a waveguide with the gradually decreasing core. A mode of the waveguide is subjected to adiabatic compression, when propagating to the tip. Thus it reaches more favorable conditions for coupling to the nanowaveguide. The tapering angle and the rate of compression determine the coupling efficiency and depend on the properties of materials. Therefore, further classification of the tapered waveguides follows the material properties:

(1) fully dielectric tapers having the dielectric core and the dielectric cladding (Figure 6(a)),

(2) hybrid tapers having the metallic core and dielectric cladding or the dielectric core and metallic cladding (Figure 6(b)),

(3) metamaterial tapers having the structured metaldielectric composite core (Figure 6(c)).

4.1. Dielectric Core and Cladding. If one takes an optical fiber, heats it up, and then pulls, the diameters of the core and shell simultaneously decrease. So it is possible to draw the core down to a nanosize diameter. Low-loss tapered fibers were fabricated and measured [18]. The optical losses for a fiber of diameter $d=750 \mathrm{~nm}$ at $\lambda=1.55 \mu \mathrm{m}$ are $0.017 \mathrm{~dB} / \mathrm{mm}$. However, it was also theoretically shown [19] that even an ideal fiber does not allow the substantial core thinning, since the propagating mode completely vanishes for the core size of one order of magnitude smaller than the wavelength. In the real fibers with imperfections this limitation is even tougher.

Nevertheless, the dielectric tapered waveguide coupler finds useful applications. As L. Zimmermann reported at the Silicon Photonics Workshop in 2011 [20], an inverted taper is commonly used for lateral coupling from a high numerical aperture lensed optical fibers with a spot size of $3 \mu \mathrm{m}$ to a $200 \times 500 \mathrm{~nm}^{2}$ silicon waveguide. The inverted taper is also used for coupling to and from the slow light photonic crystal waveguide [21] reducing the insertion loss value to $10 \mathrm{~dB}$. Tapering came out to be useful to outcoupling efficiency increase and far-field shaping of the nanopillar single photon sources $[22,23]$. The coupling efficiency can be very large ( $80 \%$ and higher) for certain pairs waveguides, for example, for silicon ridge and silicon slot waveguides [24, 25].

4.2. Metallic Core or Cladding. Tapered fiber tips covered with metal are the essential part of the scanning nearfield optical microscopy. It allows spatial compression of light below the diffraction limit by employment of SPPs [26]. Metal-dielectric-metal tapered plate waveguides were theoretically and experimentally demonstrated for the terahertz [27-30] and optical ranges [31-34] showing the ability of ultrahigh energy concentration. In the latter work [34] the maximal transmittivity of $8 \%$ was measured for the wavelength $\lambda=780 \mathrm{~nm}$ in an adiabatically tapered metalized 


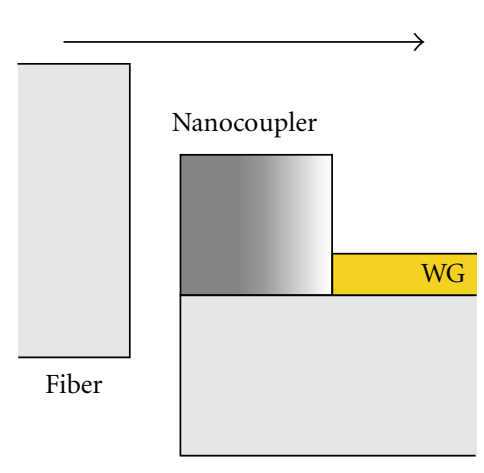

(a)

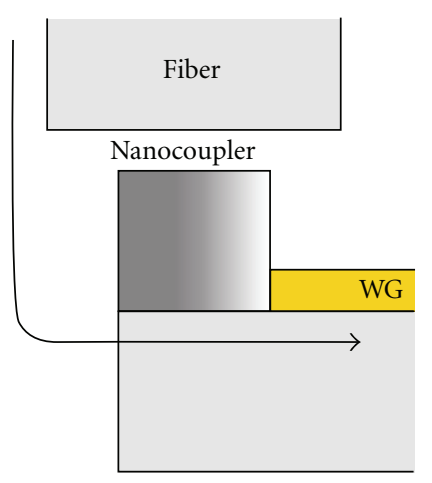

(b)

FIgure 5: Two practically important coupling configurations: (a) lateral and (b) vertical. Black arrows show the direction of light propagation.

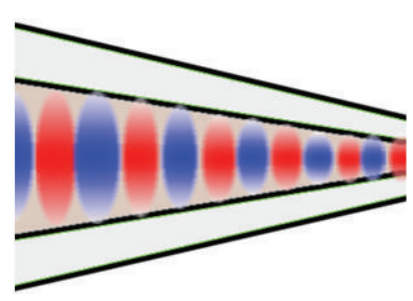

(a)

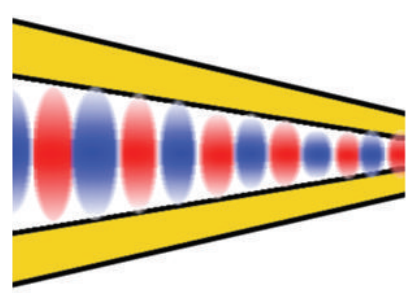

(b)

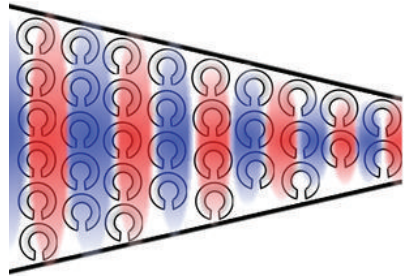

(c)

FIGURE 6: The types of taper couplers: (a) fully dielectric taper, (b) hybrid taper with metal core or cladding, (c) metamaterial taper.

fiber with the tip opening of about $150 \mathrm{~nm}$. The prominent property of such system is that a metal-dielectric-metal waveguide does not have a cut-off, and only losses limit its performance. Nanofocusing with a V-shaped metallic groove, which is a similar system, was experimentally demonstrated [35]. A wave with $\lambda=1.5 \mu \mathrm{m}$ wavelength was focused into a spot of $\lambda / 40$. The reported energy efficiency was $50 \%$. In 2004 M. Stockman predicted that a tapered metallic wire allows the giant energy accumulation and intensity enhancement at the tip of the metal taper [36]. The idea is applicable not only in the optical range but also in the terahertz range if the surface of the tapered wire is corrugated [37]. An experimental realization of the tapered wire structure was reported [38]. In this case light with $\lambda=$ $1.55 \mu \mathrm{m}$ was coupled to a $2000 \mathrm{~nm}$ wide wire and compressed down to $90 \mathrm{~nm}$. The transmittivity of $20 \%$ was shown.

Another approach was proposed in [35]. A metallic funnel consisting of long metallic nanocylinders of the radii from 10 to $24 \mathrm{~nm}$ separated with a $2 \mathrm{~nm}$ thick dielectric was shown to focus a Gaussian beam of $200 \mathrm{~nm}$ full width into a spot of $20 \mathrm{~nm}$ with a power transmittivity of $80 \%$ [39]. However, a practical realization of such system is hardly possible at the moment.

4.3. Metamaterial Core. The idea behind this nanocoupler type is to use a metamaterial as the taper core. The advantage of utilization of metamaterials is that their properties are not strictly limited to that we have from natural materials and can be designed to reach amazing diversity and values. An optical funnel containing a metal-dielectric photonic crystal was proposed in [40]. The field compression down to $\lambda / 30$ with a transmission of $13 \%$ was reported. The metamaterial-based nanotips for field enhancement were theoretically proposed $[41,42]$. The nanotip consists of metallic nanospheres of a gradually changing density. They can be used for field concentration (200 times field enhancement was reported) and the light compression to a spot of $10 \mathrm{~nm}$. In the work [41] it is stated that the power efficiency of such metamaterial tip is larger than of a metalized taper with the $10 \mathrm{~nm}$ output hole, but the exact values of the transmittivity are not mentioned.

4.4. Summary. A dielectric adiabatically tapered fiber is a well-known instrument of field concentration. However, it can provide either small spot size with low transmission or high transmission at the cost of large size of the light beam and the coupler itself. The metal- or metamaterial-based tapers can be of significantly smaller length. They can give field concentration and reasonable transmittivity (e.g., 20\%). The difficulties in practical realization of effective design solutions are connected with the technological constrains; for example, fabrication of a regularly packed bundle of metallic cylinders of radius $10 \mathrm{~nm}$ separated with $2 \mathrm{~nm}$ of dielectric is currently out of reach. 


\section{Direct Coupler}

Based on the geometrical position of one waveguide with respect to another, the direct couplers can be divided into the following classes:

(1) one waveguide next to or inside another (Figure 7(a)),

(2) end-fire direct connection of the waveguides (Figure 7(b)),

(3) resonant stub between the waveguides (Figure 7(c)).

Direct couplers as we classified them are either directional couplers or schemes including end-fire coupling. Directional couplers are classical components of photonic integrated circuits. They are typically used for wavelength division or light switching. The principle of their operation is based on the coupling between parallel waveguides due to overlapping of their modes fields. The coupling leads to the hybrid modes (supermodes, compound modes) formation. The strength of the mode coupling can be controlled by the distance between the cores. Therefore, such couplers require preliminary feeding of a large waveguide at an intermediate step. Then disposing the first waveguide in a close proximity with a nanowaveguide we initiate their coupling with a consequent transit of the light energy in the second channel. Directional couplers are characterized by the coupling length, the distance, on which the maximal amount of the energy is transferred in the second channel and vice versa. The coupling length, in turn, is proportional to the wavenumbers mismatch. As a rule, directional couplers have a relatively large coupling length, what results in the total size of such systems about 10-20 $\mu \mathrm{m}$.

The end-fire coupling scheme also exploits the fields overlapping mechanism. Here the coupling appears as a result of the field profiles matching. The scheme does not require a lengthy coupling part; however, short sizes come at a price of lower coupling efficiency. To improve the efficiency a resonant stub is often placed between the input and nanosized waveguides.

5.1. One Waveguide Next to or inside Another. A typical scheme for direct coupling is to arrange one waveguide above another such that the eigenmodes of the waveguides hybridize, and the waveguides become coupled. In such case energy from the input mode is transferred from one waveguide to another and back. Making the overlap region equal to the coupling length it is possible to achieve the maximal energy transfer efficiency. Such systems were proposed, simulated, and experimentally realized for dielectric [43] and plasmonic long- and short-range waveguides [44-48]. The coupling length may vary from several micrometers $[47,49]$ to hundreds of micrometers [43]. For certain waveguides (dielectric to long-range surface plasmon polariton) the coupling efficiency can be extremely large. The theoretical prediction of coupling efficiency CE $=60 \%$ from dielectric to plasmonic slot waveguide at $\lambda=1.55 \mu \mathrm{m}$ was confirmed in measurements [47]. However, we should take into account that light should be first coupled from a fiber to an input (silicon) waveguide and that the maximal coupling efficiency is in the order of $60-70 \%$. That makes the total coupling efficiency of the system about $30-40 \%$. It is possible to obtain light coupling by placing metal nanowires on top of the dielectric waveguide perpendicular to the latter [50]. In such arrangement the overlapping region is extremely small, and the coupling efficiency of such system is reported to be only $1 \%$.

The configuration when one waveguide protrudes into another is possible to certain waveguide types only, since the bigger waveguide should contain empty space inside. For example, it is possible to insert a silicon waveguide inside a plasmonic slot waveguide, but not into another silicon waveguide. Several designs for telecom wavelengths were proposed [51-53] and experimentally realized, giving a theoretical coupling efficiency of $88 \%$ and measured coupling efficiency about 35\% [53].

5.2. End-Fire Coupling. The end-fire coupling is the simplest case of the waveguides connections, and it is common in the optical communication systems. Nevertheless, the end-fire coupling from a dielectric to plasmonic slot waveguide or a nanowire is not usually very efficient due to the pronounced impedances mismatch. However, if the optimization of the geometry is conducted the coupling efficiency more than $70 \%[54,55]$ and even $90 \%$ can be achieved [56, 57]. For example, at the wavelength of $\lambda=1.55 \mu \mathrm{m}$ the coupling efficiency of $80 \%$ was measured for a long-range surface plasmon polaritons waveguide connected to a silicon waveguide [58]. In another work the coupling from a silicon to plasmonic slot waveguide with $30 \%$ efficiency at $\lambda=1.55 \mu \mathrm{m}$ was experimentally demonstrated [59]. End-fire coupler based on optical tunneling can be used for identical waveguides separated with a small gap [60]. Varying the width of the gap one can tune the transmission and reflection in a wide range.

5.3. Resonant Stub between the Waveguides. The use of resonant stubs (e.g., a $\lambda / 4$-transformer) for the efficient wave coupling is the well-known technique in the microwave waveguides engineering. It is based on the resonant transmission increase due to the constructive interference similarly to the antireflection coatings for lenses. The same concept can be used in the optical range, for the plasmonic waveguides of different cross-sections [11, 61-63] and for the silicon and plasmonic waveguides [64] matching. The coupling efficiency can be enhanced in comparison with the end-fire coupling. However, as based on the resonant phenomena the scheme has limited bandwidth and very individual application range.

The $\lambda$ /4-transfomer matching $500 \mathrm{~nm}$ and $50 \mathrm{~nm}$ wide plasmonic transmission lines with the coupling efficiency of $86 \%$ was shown numerically [61]. Matching of a $300 \mathrm{~nm}$ wide silicon waveguide with a $40 \mathrm{~nm}$ wide plasmonic slot waveguide with the coupling efficiency of $88 \%$ was also demonstrated [64].

5.4. Summary. While giving high values of the coupling efficiency (in case of a directional coupler up to 60\%) and 


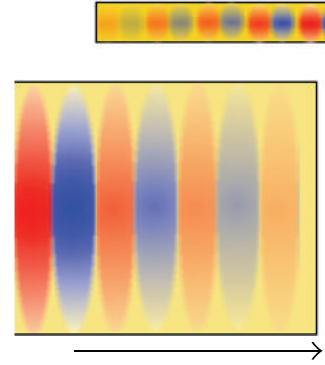

(a)

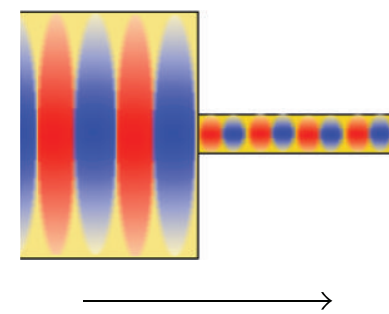

(b)

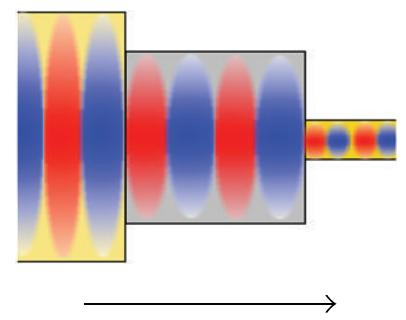

(c)

FIGURE 7: Types of the direct couplers: (a) one waveguide next to or inside another, (b) end-fire coupling, (c) resonant stub between waveguides.

being feasible for fabrication, the direct nanocouplers require additional structures such as an input silicon waveguide and a preliminary coupler to this silicon waveguide. That makes the total CE of up to $40 \%$ and extends the nanocoupler dimensions up to several dozens of micrometers. Moreover, additional structures (e.g., a preliminary coupler) may require additional processing steps during fabrication.

\section{Lens Coupler}

Lens is a well-known focusing device. However, we should emphasize that the requirements for a nanocoupler are stricter than to a focusing device, since nanocoupler should provide, apart from focusing, the high coupling efficiency. To be implemented as a nanocoupler a lens must have high transmission and execute matching of the focused beam to the nanowaveguide mode.

Based on the lens material and their functionality we divide lenses into the following categories:

(1) dielectric lens (Figure 8(a)),

(2) plasmonic lens (Figure 8(b)),

(3) negative refractive index lens (Figure 8(c)),

(4) photonic crystal lens (Figure 8(d)),

(5) hyperlens (Figure 8(e)).

6.1. Dielectric Lens. A dielectric lens is a focusing device known for several centuries. It is well described in classical optical textbooks (see, e.g., [65]). The lens can provide the excellent light transmission, but its resolution is diffraction limited and for coherent light cannot be better than $0.77 \lambda / \mathrm{NA}$, where NA is a numerical aperture. The numerical aperture cannot exceed the refractive index $n$ of a surrounding material. Even such resolution is hard to achieve in practice, as it requires a complex optical setup with a high numerical aperture objective; therefore, a conventional dielectric lens is not a suitable solution for nanocoupling.

Except a standard dielectric lens we should mention also two other focusing devices, namely, a Fresnel lens and a graded index (GRIN) lens. The Fresnel lens and zone plate are well described in optical textbooks [65]. The zone plate consists of a set of concentric rings (Fresnel zones). Rings are transparent and nontransparent in the alternating order or have the $180^{\circ}$ phase difference for the transmitted light. An important requirement is that the geometrical sizes of the zones impose the constructive interference conditions in the desired focal point. Being much lighter and much more compact than a bulk dielectric lens, the Fresnel lenses have worse resolution due to the diffraction at the zones' borders.

The GRIN lens was developed for photonics packaging [66]. It is called a lens even though its shape is far from being concave or convex (etymologically "lens" means to be of a double convex shape). It is usually a multilayer dielectric structure with a gradually changing refractive index. The refractive index gradient forces the wave propagating along the waveguide to refract and concentrate in a thin bottom layer, from where it is easier to launch it into a smaller waveguide. For example, a GRIN lens for silicon waveguides [67] allows for 45\% coupling efficiency.

Very useful for practical purposes is a focusing optical fiber that combines mechanical flexibility of a fiber with the focusing effect of a lens. Commercial focusing fibers can provide a focal spot down to $2 \mu \mathrm{m}$ in diameter [68]. The focusing effect of the fiber can be reached either by making a lensed output end or by the gradual index distribution within the fiber [69]. The extension of the latter approach is the nanoengineered fiber core [70] that uses complex refractive index distribution with the nanometric features to reach the desired functionalities. The disadvantage of the GRIN lens is that, being composed of dielectrics, it cannot overcome the diffraction limit.

6.2. Plasmonic Lens. Using plasmonic effects in metaldielectric zone plates is quite new since fabrication of fine metallic structures has become possible only recently. The plasmons, excited in the concentric grating by an incident light wave, contribute to the energy transfer to the central ring. The wave constructive interference condition should be satisfied not only for the diffracted light waves but also for the plasmons. A comprehensive review of the plasmonic lenses can be found in [71]. An interesting property of the plasmonic Fresnel lens is that different wavelengths have different focal points. That gives opportunity to use such lenses for spectroscopy purposes. 


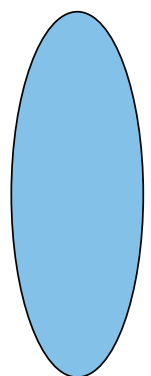

(a)

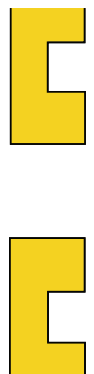

(b)

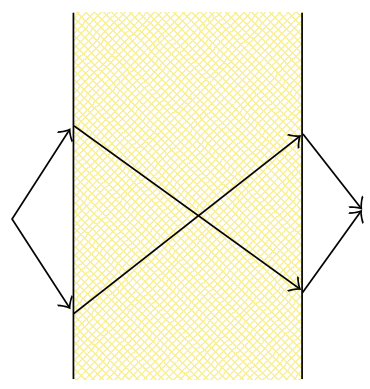

(c)

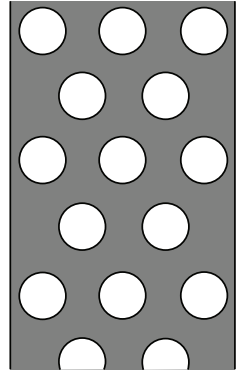

(d)

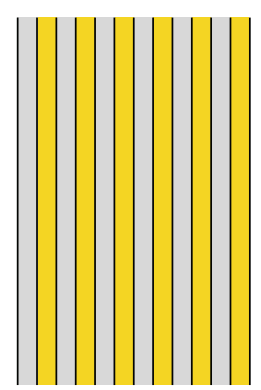

(e)

Figure 8: Types of lens couplers: (a) dielectric lens, (b) plasmonic lens, (c) negative index lens, (d) photonic crystal lens, (e) hyperlens.

The focusing effect of a plasmonic lens was experimentally shown in 2002 by Lezec et al. [72]. The theory and performance dependence on the geometry were discussed in the works [73-75]. A lens consisting of concentric rings of holes (the so-called nanopinhole lens) was numerically analyzed in [76]. Such lens allows for high transmission at $\lambda=$ $550 \mathrm{~nm}$ with focal spot of $250 \mathrm{~nm}$, that is, just a bit less than a half of the wavelength. A subwavelength focusing of the $\lambda=532 \mathrm{~nm}$ monochromatic light into the spot of $\lambda / 10$ with the transmittivity of $30 \%$ was numerically shown in [77]. Such high transmittivity is reached by adding a resonator to the entrance of the lens. Experimental investigation of the subwavelength focusing was also conducted [78]. Focusing of the visible light wave with $\lambda=633 \mathrm{~nm}$ to a subwavelength spot with the diameter $468 \mathrm{~nm}$ was reported.

As can be seen from the previously mentioned results the plasmonic lens can provide the subwavelength focusing, but practically the focal spot is not much smaller comparing to a dielectric lens, while the transmittivity is lower due to the presence of metal elements.

6.3. Negative Refractive Index Lens. The seminal idea that a plane slab of a negative index material may focus light was mentioned by Veselago in 1968 [79]. However, the interest to the negative index material lenses exploded only at the beginning of the 21st century, when Pendry showed that a negative index slab not only focuses the propagating waves, but also enhances the evanescent waves, which contain the deep-subwavelength image details [80]. Such perfect lens works in the near-field regime and can provide the ideal image that repeats all small details of an original object. To function as a superresolution lens the negative index material should be isotropic. Some special cases of anisotropic materials without spatial dispersion may also be suitable [81].

The negative refractive index occurs as a rule in materials that simultaneously possess negative dielectric permittivity and magnetic permeability. A comprehensive overview of the negative index metamaterials can be found in $[82,83]$.

A negative index slab lens can be used not only for focusing and imaging but also as a coupler. The idea to use a flat negative index slab for coupling between two identical nanowaveguides was proposed by Degiron et al. [84]. By making a concave lens out of NIM, one can match two waveguides with different cross-section. However, to be utilized for the nanocoupler construction the negative refractive index material must have bulk isotropic optical properties with small losses. The requirement for the bulk behavior means that the effective properties of the homogenized metamaterial should not depend on the slab thickness. It is usual situation that the properties of a metamaterial depend on the thickness (number of monolayer) due to the coupling between monolayers [85] with rare exceptions only [86].

In the most cases proposed metamaterials consist of planar layers, since their fabrication is based on the planar technology. That results in the optical anisotropy. To overcome such drawback several isotropic NIMs designs were proposed [87-93], but the material parameters needed to obtain the desired resonances in the optical regime can hardly be found in nature, thus limiting the application of such design to the microwave region. Moreover, the unit cell of most metamaterials is not very small comparing with the wavelength of light (usually it is in the order of $\lambda / 10--\lambda / 4$ ) that leads to spatial dispersion and hence deterioration of the effective properties introduction. So even the highest possible cubic symmetry of the unit cells and placement of unit cells in a cubic lattice do not ensure optical isotropy of negative index metamaterials [94].

The losses in a negative index metamaterial coupler are an important issue, since they reduce the coupling efficiency and decrease the spatial resolution. This is why a lot of efforts are applied at the moment to compensate the losses with gain material [95-97] or by switching for better plasmonic materials $[98,99]$. Moreover, as it is shown in recent work [100], although the subwavelength resolution is possible in metamaterial-based lenses, practical focusing beyond the diffraction limit is challenging, and when designing such device one should consider "granularity, degree of isotropy and transverse size of the metamaterial lens." Large losses, low coupling efficiency, anisotropy, and fabrication difficulties prevent a practical realization of the negative index metamaterial lens nanocoupler.

6.4. Photonic Crystal Lens. The negative refraction phenomenon may occur not only in negative index materials but also in photonic crystals typically at frequencies close to the bandgap edge [101]. This effect can be exploited to make a photonic crystal slab working for light focusing. For example, 
in the theoretical work [102] there was reported that a photonic crystal in the so-called canalization regime provides the subwavelength focusing down to a spot of $\lambda / 6$. To increase the transmission through a photonic crystal lens an antireflection coating can be applied [103]. Experimental realization of an InGaAsP/InP photonic crystal lens for $\lambda=1.5 \mu \mathrm{m}$ was reported in the work [104]. They managed to obtain a focal spot of the area $0.12 \lambda^{2}$ that overcomes the diffraction limit. Similar results for $\lambda=1.55 \mu \mathrm{m}$ in a $\operatorname{InP} / \operatorname{InGaAsP} / \operatorname{In} P$ photonic crystal lens were obtained in the work [105], where a focal spot of $0.38 \lambda$ size was demonstrated.

6.5. Hyperlens. Another solution for the subwavelength focusing involves a material with the hyperbolic dispersion, a so-called indefinite medium $[106,107]$ that simultaneously possesses positive and negative principle components of the permittivity tensor. This results in a hyperbolic isofrequency diagram, not circular or elliptical ones as in the case of conventional dielectric with positive permittivity. An important advantage of the hyperbolic material is that for a certain permittivity tensor it allows propagating waves with any values of the tangential component of the wavevector.

A medium with the hyperbolic dispersion is anisotropic by definition. To reach positive and negative permittivity for different directions one may use either metallic wires or a metal-dielectric multilayer stack. In case of wires the effective permittivity can be negative for electric field polarization along the wires, while for the polarization perpendicular to the wires the relevant principle value of the permittivity tensor is positive. In case of the metal-dielectric stack, the electric field polarized parallel to the metallic plates experiences negative dielectric response, while the permittivity associated with the perpendicular polarization is positive. A detailed theory of the multilayer and wire-medium hyperlens can be found in the works $[108,109]$.

The first theoretical work on a wire medium hyperlens [110] showed a subwavelength resolution $\lambda / 6$ for $\lambda=1.5 \mu \mathrm{m}$. In another theoretical work [109] the $\lambda / 10$ resolution was achieved in the infrared range. The same devices were successfully simulated and experimentally validated in the microwave range [111-113]. In the work [113] an impressive $\lambda / 15$ resolution was experimentally demonstrated.

Arranging metal wires in the tapering-up-like manner, not only the 1:1 image transfer but also the image magnification can be achieved. An analysis of the homogeneous medium approximation eligibility for the wire medium superlens was conducted in work [114]. In the work [115] a lens for color imaging in the visible range is proposed. The idea is to engineer the wavelength selective response introducing gaps in metallic nanowires. The experimental demonstration of a wire lens for the optical range [116] and for telecom $\lambda=1.55 \mu \mathrm{m}$ has been recently shown [117]. The resolution measured with a scanning near-field optical microscope was about $\lambda / 4$.

A flat metal-dielectric stack can transfer the subdiffraction image as a near-field lens. Using cylindrical or spherical multilayer system the image may be magnified up to above the diffraction limit. Such magnified image may be registered with an optical microscope afterwards. So the hyperlens can serve as an addition to the standard optical microscope or photolithographic system improving the resolution below the diffraction limit. The first theoretical work [118] showed the resolution of $\lambda / 4.5$. The hyperlens theory was described in paper [108]. The experimental realization of the lensing effect for the ultraviolet light $\lambda=365 \mathrm{~nm}$, which is a standard wavelength for the optical lithography, with the resolution less than $\lambda / 4$ was presented [119]. In theory the resolution was pushed further significantly. Ultraviolet light designs providing $\lambda / 18$ [120] and $\lambda / 60$ [121] resolution have been recently proposed.

Despite the fantastic resolution, the hyperlens typically has low transmission. Using the Fabry-Perot effect it is possible to increase the transmittivity values. For example, in the work [122] the three-layer thick hyperlens showed 50\% transmission. However, the magnification of such hyperlens was very low due to the small ratio of the outer and inner radii-about 1.25. Making the thickness of the hyperlens thicker, one would lose a lot in transmission. So, when designing a hyperlens one should find a trade-off between tight focusing and high transmission.

6.6. Summary. An imaging device such as lens does not necessarily provide high coupling efficiency since the primary goal of imaging is focusing of light, regardless of the transmittivity. The dielectric lens can give high transmittivity (close to 100\%) but the focal spot size is diffraction limited. A photonic crystal lens can indeed provide focusing at a specific frequency. However, the resolution of such lens is not much better than the diffraction limit allows. The plasmonic lens can provide a better spatial resolution with the cost of lower transmittivity. The negative index metamaterial lens is still far from the practical realization. From the resolution point of view, the hyperlens is the best. Theoretically it can provide the resolution as small as $\lambda / 60$. However, the transmittivity through the hyperlens is not high due to the employment of a metal-dielectric multilayer stack or metallic nanowires, which hinder its coupling applicability.

\section{Scatterer Coupler}

The main idea behind the scatterer coupler is that there are single or multiple particles that first capture the radiation from the free space and then launch it into the waveguide. Based on the geometrical placement and material we singled out the following types:

(1) antenna coupler (Figure 9(a)),

(2) grating coupler (Figure 9(b)),

(3) random scatterers (Figure 9(c)).

7.1. Antenna Coupler. According to the definition [123, 124], an antenna is a device that converts a free propagating radiation into the localized power and vice versa. For example, television antennas capture the waves propagating in air and transform them into an electrical current. In other words, an 


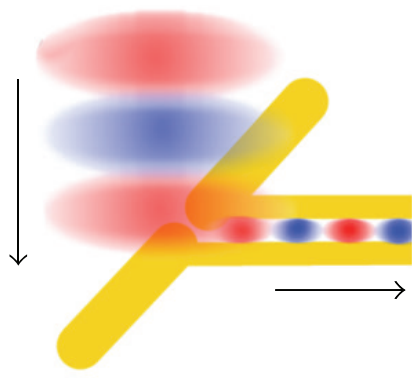

(a)

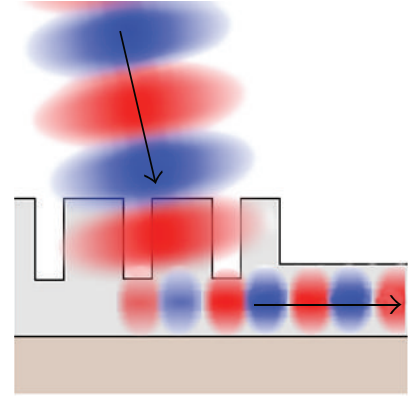

(b)

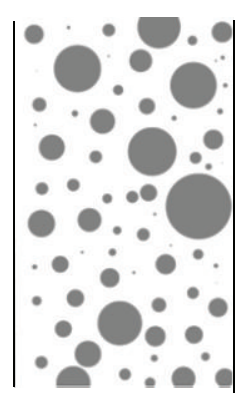

(c)

FIGURE 9: Types of scatterer couplers: (a) antenna, (b) grating, (c) random scatterers.

antenna is a coupler that matches together the impedances of free space and a waveguide. This application of antennas accounts for more than one hundred years, since Hertz, Popov, and Markoni invented the principles of the radio transmission. Now radio and microwave antennas are wellknown engineering systems.

In principle, according to the definition, some nanocouplers of other types, for example, a lens coupler, can also be called antennas. In this section, however, we limit ourselves to the traditional geometrical antenna configurations, which consist of a single of multiple metallic particles, specially tuned for accepting electromagnetic radiation.

Optical nanoantennas drew the attention of many research groups [142-151]. Nanoantennas can be considered as the optical analogue of the microwave and radio antennas [142]. An incident electromagnetic wave excites charges oscillations along the metallic antenna, which are in the optical range nothing else than localized surface plasmons, and then couples to a mode of the connected waveguide.

Despite a lot of similarities, there are essential differences between radio and plasmonic antennas [152]

(1) Metals are not such good conductors in the optical range as on the radio frequencies. Their permittivity is dispersive. It can be approximated by the Drude or more accurate Drude-Lorentz formulas [3] accounting for interband transitions in noble metals.

(2) A typical penetrations depth is several dozens of nanometers [3], and that is very important for metallic nanostructures of comparable size.

(3) The usual condition for the resonance of radiofrequency antennas is that the length of the antenna must be equal to the integer of a half of the wavelength. This condition is not satisfied for the optical antennas and should be corrected $[145,153,154]$.

Plasmonic nanoantennas have attracted huge attention in the recent years because of their ability to concentrate light in the tiny gaps and significantly enhance light intensity [143, $144,149,154]$. A comprehensive overview of the nanoantennas theory and applications can be found in $[123,152,155]$.

Despite the fact that from the very beginning antennas served for coupling to a waveguide (transmission line), the application of an antenna for optical nanocoupling was proposed only recently [125-127, 129-131]. The reasons for that are mainly the difficulties of ultrafine metallic structures fabrication (feature size on the order of 10-100 nm).

The advantage of the nanoantenna is that it is very compact. Moreover, the directivity of the antenna coupler can be tuned by design, thus enabling the maximal coupling efficiency at any desired angle of incidence. The first nanoantenna couplers analysis showed theoretically the coupling efficiency of $10 \%$ [125] for $\lambda=1.55 \mu \mathrm{m}$ and $28 \%$ [126] for $\lambda=830 \mathrm{~nm}$ for a focused Gaussian spot. Experimentally measured coupling efficiency of $15 \%$ for $\lambda=1.55 \mu \mathrm{m}$ was demonstrated in the work [129]. It was shown that the coupling efficiency can be increased at least by two times by applying additional reflectors and arranging nanoantennas in a parallel or serial array [130].

7.2. Grating Coupler. An array of nanoantennas is closely related to the diffraction grating employment as a coupler. However, the difference is that each nanoantenna has a specific length designed to be in resonance with an incident electromagnetic wave, while in the diffraction grating each line can be very long. The lines of the diffraction grating lie on top of the waveguide and scatter light into the waveguide. It is of outmost importance that the scattered radiation from each line contributes constructively to the wave propagating in the waveguide. In other words, the role of the grating is to match the tangential wavevector component $k_{t}$ of the incident wave with the waveguide propagation constant $\beta$. The grating with a specific period $P$ allows light to diffract to a diffraction order $m$ such that $\beta=k_{t}+2 \pi m / P$.

A diffraction grating is typically employed for coupling to a silicon waveguide in the vertical coupling configuration. The spot size from a single mode fiber is usually about $10 \mu \mathrm{m}$. The wave should not be strictly perpendicular to the waveguide, but incident at a small angle (about 5 degrees) to provide the wavevector matching. The maximal coupling efficiency of $60-70 \%$ was reported $[132,133]$. There was also claimed a high-efficiency (96\%) vertical coupler based on the subwavelength grating [134]. Making the grating lines concentric and adding tapering it is possible to couple and focus light simultaneously. For example, a $40 \mu \mathrm{m}$ size focusing grating coupler with $25 \%$ coupling efficiency at $\lambda=2.75 \mu \mathrm{m}$ was demonstrated in the work [135]. 
TABLE 1: Comparison of various nanocoupling approaches.

\begin{tabular}{lccccc}
\hline Approach & $\begin{array}{c}\text { Coupling } \\
\text { efficiency: low } \\
(<10 \%), \text { medium } \\
(10-50 \%) \text {, high } \\
(>50 \%)\end{array}$ & $\begin{array}{c}\text { Size: compact } \\
(<10 \mu \mathrm{m}) \text { or large } \\
(>10 \mu \mathrm{m})\end{array}$ & $\begin{array}{c}\text { Subwavelength } \\
\text { coupling or } \\
\text { focusing }\end{array}$ & $\begin{array}{c}\text { Lateral or } \\
\text { vertical } \\
\text { coupling }\end{array}$ & References \\
\hline 4.1. Tapered dielectric & High & Both & No & Lateral & {$[18-25]$} \\
4.2. Tapered metal & Medium and large & Compact & Yes & Lateral & {$[26-39]$} \\
4.3. Tapered & Low and medium & Compact & Yes & Lateral & {$[40-42]$} \\
metamaterial & High & Both & Yes & Lateral & {$[43-53]$} \\
5.1. Next to another & High & Compact & No & Lateral & {$[54-60]$} \\
5.2. End-fire & High & Compact & Yes & Lateral & {$[11,61-64]$} \\
5.3. Resonant stub & High & Large & No & Lateral & {$[66,67,69$,} \\
6.1. Dielectric lens & Medium & Compact & Yes & Lateral & {$[70]$} \\
6.2. Plasmonic lens & N/a & N/a & Yes & Lateral & {$[79,80,84$,} \\
6.3. Negative index lens & N/a & Compact & Yes & Lateral & {$[101-105]$} \\
6.4. Photonic crystal lens & Low or medium & Compact & Yes & Lateral & {$[107-122]$} \\
6.5. Hyperlens & Medium & Compact & Yes & Both & {$[125-131]$} \\
7.1. Antenna & High & Large & No & Vertical & {$[132-140]$} \\
7.2. Grating & Low & N/a & Yes & Both & {$[141]$} \\
7.3. Random scatterers & & &
\end{tabular}

A grating coupler can also be used for the surface plasmon polaritons waveguide excitation. The coupling efficiency up to $68 \%$ was theoretically shown [51, 136-138]. The grating can be designed to be polarization independent [139]. It can also combine the functions of a coupler and a nonlinear higher-order harmonics generator [140].

7.3. Random Scatterers. As we mentioned before the nanocoupler can be understood as a mode transforming device. In principle we can designate two extreme cases for mode conversion: evolution, that is, a careful adiabatic compression towards mode profile matching-this is realized by long tapered fibers-and revolution, that is, complete mode structure destruction and then construction of another mode in the same way as a new building can be built from the bricks of a ruined house. This analogy would mean introduction of a set of random scatterers. The photons coming from the first waveguide experience multiple scattering, and statistically some of them can couple to a mode of the second waveguide.

It is clear that the coupling efficiency of such random material coupler cannot be high due to the random nature of the photon scattering process. However, there was recently shown that under certain circumstances a disordered medium can work for light focusing [141].

7.4. Summary. The antenna nanocoupler is a natural transition of a standard microwave approach for coupling an electromagnetic wave to an optical (plasmonic) waveguide. There is a theoretical $50 \%$ limit of the coupling efficiency of the antenna systems [156] due to reradiation of the captured power back into free space. Practically, the nanoantennas can exhibit the coupling efficiency close to the theoretical limit, being constrained only by optical losses and fabrication imperfections. Nevertheless, the main advantage of the nanoantenna coupler is that it is the most compact among all the nanocoupling solutions. Diffraction gratings are very efficient vertical coupling solutions providing the coupling efficiency up to $70 \%$. Their disadvantage is relatively large size (more than $10 \mu \mathrm{m}$ ). The random scatterers have very low coupling efficiency due to stochastic scattering of light into the desired waveguide mode, so they are hardly suitable for the nanocoupling applications.

\section{Other Solutions}

In this section we included all other ideas that do not fall into the previously mentioned categories. Such coupling ideas are:

(1) transformation optics coupler,

(2) topology optimization designed coupler.

8.1. Transformation Optics Coupler. Inspired by the metamaterials possibilities of obtaining whatever permittivity and permeability values the field of transformation optics has recently emerged [157-159]. The transformation optics solves the problem of determination of the spatial permittivity and permeability distribution that provides a required wave propagation trajectory. For example, in case of invisibility cloaking engineering it is required that light rays pass around an object not interacting with it [160].

A problem of coupling can be expressed in the language of the transformation optics. To design a nanocoupler 
is to determine such spatial distribution of permittivity and permeability that provides impedance matching and squeezing of the light from a thick waveguide to a thin one. In some sense the GRIN lens is also an example of the transformation optics application. Some designs for squeezing light [161] or light concentration $[162,163]$ were proposed.

Very often, however, the transformation optics designs require unusual values of permittivity and permeability that are not realistic even with the help of metamaterials (e.g., diverging permittivity and permeability values without losses or extremely large anisotropy).

8.2. Topology Optimization. Another approach to the coupler design is to select from the very beginning the realistic material properties (in the simplest case, of two materials) and to determine the spatial distribution (topology) of the materials that gives the largest coupling efficiency. With the efficient numerical algorithms one has no need to go deep into physical consideration when designing the nanocoupler. Such approach is called topology optimization [164]. The optimized structures usually have very weird shapes [165]. Setting some constraints on the geometrical size of the fine features one can design an efficient coupler that is reasonable for fabrication [166].

8.3. Summary. The transformation optics devices very often require unrealistic material properties. In contrary to the transformation optics the topology optimization starts from the realistic material properties and then finds the necessary geometry. We should admit that both of these approaches can be applied to almost any coupler in the Sections 4-7. Therefore we should better say that these are not independent physical approaches, but rather useful design methodologies.

\section{Conclusions}

In this paper we have analyzed various physical principles that can be used for coupling light from an optical fiber or free space to nanosized waveguides. The range of approaches is very broad, so we divided the subject into four classes (tapered waveguide coupler, direct coupler, lens coupler, and scatterer coupler). The most important features of each approach are summarized in Table 1.

The most compact solution for the nanocoupling is the antenna coupler. The most efficient are the tapered waveguide and the grating coupler combined with the directional coupler. The hyperlens gives a good trade-off between subdiffraction imaging and transmission. The designs that use negative index materials are lossy and therefore can hardly be used for the nanocoupler at the moment. A discovery of new plasmonic materials with smaller losses or optical losses compensation with gain can probably make the latter approaches useful for light coupling.

We see the future of the nanocouplers mostly in their technical improvement. This includes a search for better materials, optimization of the designs, and fabrication technologies. The previously mentioned coupling approaches can also be the building blocks of more advanced photonic devices. For example, tapering the directional slot waveguides coupler and filling the slots with a nonlinear material [167] give a new nanophotonic functionality and can be used for all-optical switching. Another direction is the transfer of the optical coupling approaches to other fields of physics, for example, to acoustics or surface waves.

\section{Acknowledgments}

The authors acknowledge the members of the Metamaterials group at DTU Fotonik for useful discussions and the financial support from the Danish Council for Technical and Production Sciences through the projects GraTer (11116991), NIMbus and THzCOW.

\section{References}

[1] D. A. B. Miller, "Optical interconnects to electronic chips," Applied Optics, vol. 49, no. 25, pp. F59-F70, 2010.

[2] S. Kawata, M. Ohtsu, and M. Irie, Nano-Optics, vol. 84, Springer, 2002.

[3] S. A. Maie, Plasmonics: Fundamentals and Applications, Springer, 2007.

[4] S. I. Bozhevolnyi, Plasmonic Nanoguides and Circuits, Pan Stanford, 2008.

[5] R. Zia, M. D. Selker, P. B. Catrysse, and M. L. Brongersma, "Geometries and materials for subwavelength surface plasmon modes," Journal of the Optical Society of America A, vol. 21, no. 12, pp. 2442-2446, 2004.

[6] A. Boltasseva, T. Nikolajsen, K. Leosson, K. Kjaer, M. S. Larsen, and S. I. Bozhevolnyi, "Integrated optical components utilizing long-range surface plasmon polaritons," Journal of Lightwave Technology, vol. 23, no. 1, pp. 413-422, 2005.

[7] S. I. Bozhevolnyi, "Effective-index modeling of channel plasmon polaritons," Optics Express, vol. 14, no. 20, pp. 94679476, 2006.

[8] S. I. Bozhevolnyi, V. S. Volkov, E. Devaux, and T. W. Ebbesen, "Channel plasmon-polariton guiding by subwavelength metal grooves," Physical Review Letters, vol. 95, no. 4, Article ID 046802, 4 pages, 2005.

[9] E. Moreno, S. G. Rodrigo, S. I. Bozhevolnyi, L. MartínMoreno, and F. J. García-Vidal, "Guiding and focusing of electromagnetic fields with wedge plasmon polaritons," Physical Review Letters, vol. 100, no. 2, Article ID 023901, 4 pages, 2008.

[10] L. Liu, Z. Han, and S. He, "Novel surface plasmon waveguide for high integration," Optics Express, vol. 13, no. 17, pp. 66456650, 2005.

[11] G. Veronis and S. Fan, "Modes of subwavelength plasmonic slot waveguides," Journal of Lightwave Technology, vol. 25, no. 9, pp. 2511-2521, 2007.

[12] S. A. Maier, P. G. Kik, and H. A. Atwater, "Observation of coupled plasmon-polariton modes in Au nanoparticle chain waveguides of different lengths: estimation of waveguide loss," Applied Physics Letters, vol. 81, no. 9, Article ID 1714, 3 pages, 2002.

[13] M. L. Brongersma and P. G. Kik, Surface Plasmon Nanophotonics, vol. 131, Springer, 2007. 
[14] M. I. Stockman, "Nanoplasmonics: past, present, and glimpse into future," Optics Express, vol. 19, no. 22, pp. 22029-22106, 2011.

[15] D. K. Gramotnev and S. I. Bozhevolnyi, "Plasmonics beyond the diffraction limit," Nature Photonics, vol. 4, no. 2, pp. 8391, 2010.

[16] S. W. Hell and J. Wichmann, "Breaking the diffraction resolution limit by stimulated emission: stimulated-emissiondepletion fluorescence microscopy," Optics Letters, vol. 19, no. 11, pp. 780-782, 1994.

[17] E. Rittweger, K. Y. Han, S. E. Irvine, C. Eggeling, and S. W. Hell, "STED microscopy reveals crystal colour centres with nanometric resolution," Nature Photonics, vol. 3, no. 3, pp. 144-147, 2009.

[18] G. Brambilla, V. Finazzi, and D. J. Richardson, "Ultra-lowloss optical fiber nanotapers," Optics Express, vol. 12, no. 10, pp. 2258-2263, 2004.

[19] M. Sumetsky, "How thin can a microfiber be and still guide light?” Optics Letters, vol. 31, no. 7, pp. 870-872, 2006.

[20] L. Zimmermann, "State of the art and trends in silicon photonics packaging," 2011, http://www.siliconphotonics.eu/ workshop230511_slides.html.

[21] Q. V. Tran, S. Combrí, P. Colman, and A. De Rossi, "Photonic crystal membrane waveguides with low insertion losses," Applied Physics Letters, vol. 95, no. 6, Article ID 061105, 3 pages, 2009.

[22] N. Gregersen, T. R. Nielsen, J. Claudon, J. M. Gérard, and J. Mørk, "Controlling the emission profile of a nanowire with a conical taper," Optics Letters, vol. 33, no. 15, pp. 1693-1695, 2008.

[23] J. Claudon, J. Bleuse, N. S. Malik et al., "A highly efficient single-photon source based on a quantum dot in a photonic nanowire," Nature Photonics, vol. 4, no. 3, pp. 174-177, 2010.

[24] V. M. N. Passaro and M. la Notte, "Optimizing SOI slot waveguide fabrication tolerances and strip-slot coupling for very efficient optical sensing," Sensors, vol. 12, no. 3, pp. 2436-2455, 2012.

[25] H. Zhang, J. Zhang, S. Chen et al., "CMOS-compatible fabrication of silicon-based sub-100-nm slot waveguide with efficient channel-slot coupler," IEEE Photonics Technology Letters, vol. 24, no. 1, pp. 10-12, 2012.

[26] N. M. Arslanov and S. A. Moiseev, "Ultrahigh interference spatial compression of light inside the subwavelength aperture of a near-field optical probe," Journal of the Optical Society of America A, vol. 24, no. 3, pp. 831-838, 2007.

[27] A. Rusina, M. Durach, K. A. Nelson, and M. I. Stockman, "Nanoconcentration of terahertz radiation in plasmonic waveguides," Optics Express, vol. 16, no. 23, pp. 18576-18589, 2008.

[28] J. Liu, R. Mendis, and D. M. Mittleman, "The transition from a TEM-like mode to a plasmonic mode in parallel-plate waveguides," Applied Physics Letters, vol. 98, no. 23, Article ID 231113, 3 pages, 2011.

[29] K. Iwaszczuk, A. Andryieuski, A. Lavrinenko, X.-C. Zhang, and P. U. Jepsen, "Non-invasive terahertz field imaging inside parallel plate waveguides," Applied Physics Letters, vol. 99, no. 7, Article ID 071113, 3 pages, 2011.

[30] K. Iwaszczuk, A. Andryieuski, A. Lavrinenko, X.-C. Zhang, and P. U. Jepsen, "Terahertz field enhancement to the MV/cm regime in a tapered parallel plate waveguide," Optics Express, vol. 20, no. 8, pp. 8344-8355, 2012.

[31] D. F. P. Pile and D. K. Gramotnev, "Adiabatic and nonadiabatic nanofocusing of plasmons by tapered gap plasmon waveguides," Applied Physics Letters, vol. 89, no. 4, Article ID 041111, 3 pages, 2006.
[32] I.-Y. Park, S. Kim, J. Choi et al., "Plasmonic generation of ultrashort extreme-ultraviolet light pulses," Nature Photonics, vol. 5, no. 11, pp. 677-681, 2011.

[33] S. Vedantam, H. Lee, J. Tang, J. Conway, M. Staffaroni, and E. Yablonovitch, "A plasmonic dimple lens for nanoscale focusing of light," Nano Letters, vol. 9, no. 10, pp. 3447-3452, 2009.

[34] F. Renna, D. Cox, and G. Brambilla, "Efficient subwavelength light confinement using surface plasmon polaritons in tapered fibers," Optics Express, vol. 17, no. 9, pp. 76587663, 2009.

[35] H. Choi, D. F. P. Pile, S. Nam, G. Bartal, and X. Zhang, "Compressing surface plasmons for nano-scale optical focusing," Optics Express, vol. 17, no. 9, pp. 7519-7524, 2009.

[36] M. I. Stockman, "Nanofocusing of optical energy in tapered plasmonic waveguides," Physical Review Letters, vol. 93, no. 13, Article ID 137404, 4 pages, 2004.

[37] S. A. Maier, S. R. Andrews, L. Martín-Moreno, and F. J. García-Vidal, "Terahertz surface plasmon-polariton propagation and focusing on periodically corrugated metal wires," Physical Review Letters, vol. 97, no. 17, Article ID 176805, 4 pages, 2006.

[38] E. Verhagen, M. Spasenović, A. Polman, and L. Kuipers, "Nanowire plasmon excitation by adiabatic mode transformation," Physical Review Letters, vol. 102, no. 20, Article ID 203904, 4 pages, 2009.

[39] X. L. Zhou, Y. Q. Fu, S. Y. Wang, A. J. Peng, and Z. H. Cai, "Funnel-shaped arrays of metal nano-cylinders for nanofocusing," Chinese Physics Letters, vol. 25, no. 9, pp. 32963299, 2008.

[40] A. A. Govyadinov and V. A. Podolskiy, "Metamaterial photonic funnels for subdiffraction light compression and propagation," Physical Review B, vol. 73, no. 15, Article ID 155108, 5 pages, 2006.

[41] S. Mühlig, C. Rockstuhl, J. Pniewski, C. R. Simovski, S. A. Tretyakov, and F. Lederer, "Three-dimensional metamaterial nanotips," Physical Review B, vol. 81, no. 7, Article ID 075317, 8 pages, 2010.

[42] C. Rockstuhl, C. R. Simovski, S. A. Tretyakov, and F. Lederer, "Metamaterial nanotips," Applied Physics Letters, vol. 94, no. 11, Article ID 113110, 3 pages, 2009.

[43] S. Dong, H. Ding, Y. Liu, and X. Qi, "Investigation of evanescent coupling between tapered fiber and a multimode slab waveguide," Applied Optics, vol. 51, no. 10, pp. C152C157, 2012.

[44] R. Yan, P. Pausauskie, J. Huang, and P. Yang, "Direct photonic-plasmonic coupling and routing in single nanowires," Proceedings of the National Academy of Sciences of the United States of America, vol. 106, no. 50, pp. 21045-21050, 2009.

[45] Q. Li and M. Qiu, "Structurally-tolerant vertical directional coupling between metal-insulator-metal plasmonic waveguide and silicon dielectric waveguide," Optics Express, vol. 18, no. 15, pp. 15531-15543, 2010.

[46] Q. Li, Y. Song, G. Zhou, Y. Su, and M. Qiu, "Asymmetric plasmonic-dielectric coupler with short coupling length, high extinction ratio, and low insertion loss," Optics Letters, vol. 35, no. 19, pp. 3153-3155, 2010.

[47] C. Delacour, S. Blaize, P. Grosse et al., "Efficient directional coupling between silicon and copper plasmonic nanoslot waveguides: toward metal-oxide-silicon nanophotonics," Nano Letters, vol. 10, no. 8, pp. 2922-2926, 2010.

[48] R. Wan, F. Liu, Y. Huang et al., "Excitation of short range surface plasmon polariton mode based on integrated hybrid 
coupler," Applied Physics Letters, vol. 97, no. 14, Article ID 141105, 3 pages, 2010.

[49] Q. Li, S. Wang, Y. Chen, M. Yan, L. Tong, and M. Qiu, "Experimental demonstration of plasmon propagation, coupling, and splitting in silver nanowire at 1550-nm wavelength," IEEE Journal on Selected Topics in Quantum Electronics, vol. 17, no. 4, pp. 1107-1111, 2010.

[50] A. L. Pyayt, B. Wiley, Y. Xia, A. Chen, and L. Dalton, "Integration of photonic and silver nanowire plasmonic waveguides," Nature Nanotechnology, vol. 3, no. 11, pp. 660-665, 2008.

[51] Z. Wang, N. Zhu, Y. Tang, L. Wosinski, D. Dai, and S. $\mathrm{He}$, "Ultracompact low-loss coupler between strip and slot waveguides," Optics Letters, vol. 34, no. 10, pp. 1498-1500, 2009.

[52] J. Gosciniak, V. S. Volkov, S. I. Bozhevolnyi, L. Markey, S. Massenot, and A. Dereux, "Fiber-coupled dielectric-loaded plasmonic waveguides," Optics Express, vol. 18, no. 5, pp. 5314-5319, 2010.

[53] J. Tian, S. Yu, W. Yan, and M. Qiu, "Broadband highefficiency surface-plasmon-polariton coupler with siliconmetal interface," Applied Physics Letters, vol. 95, no. 1, Article ID 013504, 3 pages, 2009.

[54] S. Y. Lee, J. Park, M. Kang, and B. Lee, "Highly efficient plasmonic interconnector based on the asymmetric junction between metal-dielectric-metal and dielectric slab waveguides," Optics Express, vol. 19, no. 10, pp. 9562-9574, 2011.

[55] Y. Song, J. Wang, Q. Li, M. Yan, and M. Qiu, "Broadband coupler between silicon waveguide and hybrid plasmonic waveguide," Optics Express, vol. 18, no. 12, pp. 13173-13179, 2010.

[56] X. W. Chen, V. Sandoghdar, and M. Agio, "Nanofocusing radially-polarized beams for high-throughput funneling of optical energy to the near field," Optics Express, vol. 18, no. 10, pp. 10878-10887, 2010.

[57] X. W. Chen, V. Sandoghdar, and M. Agio, "Highly efficient interfacing of guided plasmons and photons in nanowires," Nano Letters, vol. 9, no. 11, pp. 3756-3761, 2009.

[58] R. M. Briggs, J. Grandidier, S. P. Burgos, E. Feigenbaum, and H. A. Atwater, "Efficient coupling between dielectric-loaded plasmonic and silicon photonic waveguides," Nano Letters, vol. 10, no. 12, pp. 4851-4857, 2010.

[59] Z. Han, A. Y. Elezzabi, and Van, "Experimental realization of subwavelength plasmonic slot waveguides on a silicon platform," Optics Letters, vol. 35, no. 4, pp. 502-504, 2010.

[60] D. L. MacFarlane, M. P. Christensen, K. Liu et al., "Four-port nanophotonic frustrated total internal reflection coupler," IEEE Photonics Technology Letters, vol. 24, no. 1, pp. 58-60, 2012.

[61] P. Ginzburg and M. Orenstein, "Plasmonic transmission lines: from micro to nano scale with $\lambda / 4$ impedance matching," Optics Express, vol. 15, no. 11, pp. 6762-6767, 2007.

[62] J. Liu, H. Zhao, Y. Zhang, and S. Liu, "Resonant cavity based antireflection structures for surface plasmon waveguides," Applied Physics B, vol. 98, no. 4, pp. 797-802, 2010.

[63] Ş. Kocabaş, G. Veronis, D. A. B. Miller, and S. Fan, "Modal analysis and coupling in metal-insulator-metal waveguides," Physical Review B, vol. 79, no. 3, Article ID 035120, 17 pages, 2009.

[64] A. Pannipitiya, I. D. Rukhlenko, M. Premaratne, H. T. Hattori, and G. P. Agrawal, "Improved transmission model for metal-dielectric-metal plasmonic waveguides with stub structure," Optics Express, vol. 18, no. 6, pp. 6191-6204, 2010.
[65] M. Born, E. Wolf, and A. B. Bhatia, Principles of Optics, vol. 10, Pergamon Pr, 1975.

[66] D. R. Beltrami, J. D. Love, A. Durandet et al., "Planar gradedindex (GRIN) PECVD lens," Electronics Letters, vol. 32, no. 6, pp. 549-550, 1996.

[67] T. H. Loh, Q. Wang, J. Zhu et al., "Ultra-compact multilayer $\mathrm{Si} / \mathrm{SiO}_{2}$ GRIN lens mode-size converter for coupling singlemode fiber to Si-wire waveguide," Optics Express, vol. 18, no. 21, pp. 21519-21533, 2010.

[68] “OZ Optics Ltd," http://ozoptics.com.

[69] M. D. Feit and J. A. Fleck, "Light propagation in graded-index optical fibers," Applied Optics, vol. 17, no. 24, pp. 3990-3998, 1978.

[70] J. M. Nowosielski, R. Buczynski, F. Hudelist, A. J. Waddie, and M. R. Taghizadeh, "Nanostructured GRIN microlenses for Gaussian beam focusing," Optics Communications, vol. 283, no. 9, pp. 1938-1944, 2010.

[71] Y. Fu and X. Zhou, "Plasmonic lenses: a review," Plasmonics, vol. 5, no. 3, pp. 287-310, 2010.

[72] H. J. Lezec, A. Degiron, E. Devaux et al., "Beaming light from a subwavelength aperture," Science, vol. 297, no. 5582, pp. 820-822, 2002.

[73] A. G. Curto, A. Manjavacas, and F. J. G. De Abajo, "Near-field focusing with optical phase antennas," Optics Express, vol. 17, no. 20, pp. 17801-17811, 2009.

[74] D. R. Jackson, J. Chen, R. Qiang, F. Capolino, and A. A. Oliner, "The role of leaky plasmon waves in the directive beaming of light through a subwavelength aperture," Optics Express, vol. 16, no. 26, pp. 21271-21281, 2008.

[75] L. Martín-Moreno, F. J. Garcia-Vidal, H. J. Lezec, A. Degiron, and T. W. Ebbesen, "Theory of highly directional emission from a single subwavelength aperture surrounded by surface corrugations," Physical Review Letters, vol. 90, no. 16, Article ID 167401, 4 pages, 2003.

[76] Y. Fu, C. Du, W. Zhou, and L. E. N. Lim, "Nanopinholesbased optical superlens," Research Letters in Physics, vol. 2008, Article ID 148505, 5 pages, 2008.

[77] M. Consonni, J. Hazart, G. Ĺrondel, and A. Vial, "Nanometer scale light focusing with high cavity-enhanced output," Journal of Applied Physics, vol. 105, no. 8, Article ID 084308, 6 pages, 2009.

[78] J. Wang and W. Zhou, "Experimental investigation of focusing of gold planar plasmonic lenses," Plasmonics, vol. 5, no. 4, pp. 325-329, 2010.

[79] V. G. Veselago, "The electrodynamics of substances with simultaneously negative values of $\varepsilon$ and $\mu$," Soviet Physics Uspekhi, vol. 10, no. 4, pp. 509-514, 1968.

[80] J. B. Pendry, "Negative refraction makes a perfect lens," Physical Review Letters, vol. 85, no. 18, pp. 3966-3969, 2000.

[81] N. H. Shen, S. Foteinopoulou, M. Kafesaki et al., "Compact planar far-field superlens based on anisotropic left-handed metamaterials," Physical Review B, vol. 80, no. 11, Article ID 115123, 9 pages, 2009.

[82] K. Busch, G. von Freymann, S. Linden, S. F. Mingaleev, L. Tkeshelashvili, and M. Wegener, "Periodic nanostructures for photonics," Physics Reports, vol. 444, no. 3-6, pp. 101-202, 2007.

[83] W. Cai and V. Shalaev, Optical Metamaterials: Fundamentals and Applications, Springer, 2009.

[84] A. Degiron, D. R. Smith, J. J. Mock, B. J. Justice, and J. Gollub, "Negative index and indefinite media waveguide couplers," Applied Physics A, vol. 87, no. 2, pp. 321-328, 2007.

[85] A. Andryieuski, C. Menzel, C. Rockstuhl, R. Malureanu, F. Lederer, and A. Lavrinenko, "Homogenization of resonant 
chiral metamaterials," Physical Review B, vol. 82, no. 23, Article ID 235107, 7 pages, 2010.

[86] A. Andryieuski, C. Menzel, C. Rockstuhl, R. Malureanu, and A. V. Lavrinenko, "The split cube in a cage: bulk negativeindex material for infrared applications," Journal of Optics A, vol. 11, no. 11, Article ID 114010, 2009.

[87] A. K. Iyer and G. V. Eleftheriades, "A three-dimensional isotropic transmission-line metamaterial topology for freespace excitation," Applied Physics Letters, vol. 92, no. 26, Article ID 261106, 3 pages, 2008.

[88] C. Caloz and T. Itoh, Electromagnetic Metamaterials: Transmission Line Theory and Microwave Applications: The Engineering Approach, Wiley-IEEE Press, 2006.

[89] T. Koschny, L. Zhang, and C. M. Soukoulis, "Isotropic threedimensional left-handed metamaterials," Physical Review B, vol. 71, no. 12, Article ID 121103, 4 pages, 2005.

[90] V. Yannopapas and A. Moroz, "Negative refractive index metamaterials from inherently non-magnetic materials for deep infrared to terahertz frequency ranges," Journal of Physics Condensed Matter, vol. 17, no. 25, pp. 3717-3734, 2005.

[91] I. Vendik, O. Vendik, and M. Odit, "Isotropic artificial media with simultaneously negative permittivity and permeability," Microwave and Optical Technology Letters, vol. 48, no. 12, pp. 2553-2556, 2006.

[92] A. G. Kussow, A. Akyurtlu, and N. Angkawisittpan, "Optically isotropic negative index of refraction metamaterial," Physica Status Solidi (B), vol. 245, no. 5, pp. 992-997, 2008.

[93] A. Alù and N. Engheta, "Three-dimensional nanotransmission lines at optical frequencies: a recipe for broadband negative-refraction optical metamaterials," Physical Review B, vol. 75, no. 2, Article ID 024304, 20 pages, 2007.

[94] C. Menzel, C. Rockstuhl, R. Iliew et al., "High symmetry versus optical isotropy of a negative-index metamaterial," Physical Review B, vol. 81, no. 19, Article ID 195123, 6 pages, 2010.

[95] Y. Sivan, S. Xiao, U. K. Chettiar, A. V. Kildishev, and V. M. Shalaev, "Frequency-domain simulations of a negativeindex material with embedded gain," Optics Express, vol. 17, no. 26, pp. 24060-24074, 2009.

[96] A. N. Lagarkov, V. N. Kisel, and A. K. Sarychev, "Loss and gain in metamaterials," Journal of the Optical Society of America B, vol. 27, no. 4, pp. 648-659, 2010.

[97] A. Fang, T. Koschny, M. Wegener, and C. M. Soukoulis, "Selfconsistent calculation of metamaterials with gain," Physical Review B, vol. 79, no. 24, Article ID 241104, 4 pages, 2009.

[98] A. Boltasseva and H. A. Atwater, "Low-loss plasmonic metamaterials," Science, vol. 331, no. 6015, pp. 290-291, 2011.

[99] P. R. West, S. Ishii, G. V. Naik, N. K. Emani, V. M. Shalaev, and A. Boltasseva, "Searching for better plasmonic materials," Laser and Photonics Reviews, vol. 4, no. 6, pp. 795-808, 2010.

[100] X.-X. Liu and A. Alù, "Limitations and potentials of metamaterial lenses," Journal of Nanophotonics, vol. 5, no. 1, Article ID 053509, 2011.

[101] M. Notomi, "Theory of light propagation in strongly modulated photonic crystals: refractionlike behavior in the vicinity of the photonic band gap," Physical Review B, vol. 62, no. 16, pp. 10696-10705, 2000.

[102] P. A. Belov, C. R. Simovski, and P. Ikonen, "Canalization of subwavelength images by electromagnetic crystals," Physical Review B, vol. 71, no. 19, Article ID 193105, 4 pages, 2005.
[103] W. Šmigaj, B. Gralak, R. Pierre, and G. Tayeb, "Antireflection gratings for a photonic-crystal flat lens," Optics Letters, vol. 34, no. 22, pp. 3532-3534, 2009.

[104] B. D. F. Casse, W. T. Lu, R. K. Banyal et al., "Imaging with subwavelength resolution by a generalized superlens at infrared wavelengths," Optics Letters, vol. 34, no. 13, pp. 1994-1996, 2009.

[105] M. Hofman, N. Fabre, X. Mélique, D. Lippens, and O. Vanbésien, "Defect assisted subwavelength resolution in IIIV semiconductor photonic crystal flat lenses with $n=-1$," Optics Communications, vol. 283, no. 6, pp. 1169-1173, 2010.

[106] D. R. Smith and D. Schurig, "Electromagnetic wave propagation in media with indefinite permittivity and permeability tensors," Physical Review Letters, vol. 90, no. 7, Article ID 077405, 4 pages, 2003.

[107] D. R. Smith, P. Kolinko, and D. Schurig, "Negative refraction in indefinite media," Journal of the Optical Society of America $B$, vol. 21, no. 5, pp. 1032-1043, 2004.

[108] Z. Jacob, L. V. Alekseyev, and E. Narimanov, "Semiclassical theory of the hyperlens," Journal of the Optical Society of America A, vol. 24, no. 10, pp. A52-A59, 2007.

[109] M. G. Silveirinha, P. A. Belov, and C. R. Simovski, "Subwavelength imaging at infrared frequencies using an array of metallic nanorods," Physical Review B, vol. 75, no. 3, Article ID 035108, 12 pages, 2007.

[110] J. Elser, R. Wangberg, V. A. Podolskiy, and E. E. Narimanov, "Nanowire metamaterials with extreme optical anisotropy," Applied Physics Letters, vol. 89, no. 26, Article ID 261102, 3 pages, 2006.

[111] P. A. Belov, P. Ikonen, C. R. Simovski, Y. Hao, and S. A. Tretyakov, "Magnification of subwavelength field distributions using a tapered array of wires operating in the canalization regime," in Proceedings of the IEEE International Symposium on Antennas and Propagation and USNC/URSI National Radio Science Meeting (APSURSI '08), pp. 8-11, July 2008.

[112] Y. Zhao, P. Belov, and Y. Hao, "Subwavelength internal imaging by means of a wire medium," Journal of Optics A, vol. 11, no. 7, Article ID 075101, 2009.

[113] P. A. Belov, Y. Hao, and S. Sudhakaran, "Subwavelength microwave imaging using an array of parallel conducting wires as a lens," Physical Review B, vol. 73, no. 3, Article ID 033108, 4 pages, 2006.

[114] A. Fang, T. Koschny, and C. M. Soukoulis, "Optical anisotropic metamaterials: negative refraction and focusing," Physical Review B, vol. 79, no. 24, Article ID 245127, 7 pages, 2009.

[115] S. Kawata, A. Ono, and P. Verma, "Subwavelength colour imaging with a metallic nanolens," Nature Photonics, vol. 2, no. 7, pp. 438-442, 2008.

[116] J. Yao, K. T. Tsai, Y. Wang et al., "Imaging visible light using anisotropic metamaterial slab lens," Optics Express, vol. 17, no. 25, pp. 22380-22385, 2009.

[117] B. D. F. Casse, W. T. Lu, Y. J. Huang, E. Gultepe, L. Menon, and S. Sridhar, "Super-resolution imaging using a three-dimensional metamaterials nanolens," Applied Physics Letters, vol. 96, no. 2, Article ID 023114, 3 pages, 2010.

[118] Z. Jacob, L. V. Alekseyev, and E. Narimanov, "Optical hyperlens: far-field imaging beyond the diffraction limit," Optics Express, vol. 14, no. 18, pp. 8247-8256, 2006.

[119] C. Jeppesen, R. B. Nielsen, A. Boltasseva, S. Xiao, N. A. Mortensen, and A. Kristensen, "Thin film Ag superlens 
towards lab-on-a-chip integration," Optics Express, vol. 17, no. 25, pp. 22543-22552, 2009.

[120] Y. Xiong, Z. Liu, and X. Zhang, "A simple design of flat hyperlens for lithography and imaging with half-pitch resolution down to $20 \mathrm{~nm}$," Applied Physics Letters, vol. 94, no. 20, Article ID 203108, 3 pages, 2009.

[121] Q. Meng, X. Zhang, L. Cheng et al., "Deep subwavelength focusing of light by a trumpet hyperlens," Journal of Optics, vol. 13, no. 7, Article ID 075102, 2011.

[122] J. Kerbst, S. Schwaiger, A. Rottler et al., "Enhanced transmission in rolled-up hyperlenses utilizing Fabry-Pérot resonances," Applied Physics Letters, vol. 99, no. 19, Article ID 191905, 3 pages, 2011.

[123] P. Bharadwaj, B. Deutsch, and L. Novotny, "Optical antennas," Advances in Optics and Photonics, vol. 1, no. 2, pp. 438483, 2009.

[124] C. A. Balanis, Antenna Theory, Wiley, New York, NY, USA, 1997.

[125] J. Wen, S. Romanov, and U. Peschel, "Excitation of plasmonic gap waveguides by nanoantennas," Optics Express, vol. 17, no. 8, pp. 5925-5932, 2009.

[126] J. S. Huang, T. Feichtner, P. Biagioni, and B. Hecht, "Impedance matching and emission properties of nanoantennas in an optical nanocircuit," Nano Letters, vol. 9, no. 5, pp. 1897-1902, 2009.

[127] Z. Fang, Y. Lu, L. Fan, C. Lin, and X. Zhu, "Surface plasmon polariton enhancement in silver nanowire-nanoantenna structure," Plasmonics, vol. 5, no. 1, pp. 57-62, 2010.

[128] Z. Fang, L. Fan, C. Lin, D. Zhang, A. J. Meixner, and X. Zhu, "Plasmonic coupling of bow tie antennas with Ag nanowire," Nano Letters, vol. 11, no. 4, pp. 1676-1680, 2011.

[129] J. Wen, P. Banzer, A. Kriesch, D. Ploss, B. Schmauss, and U. Peschel, "Experimental cross-polarization detection of coupling far-field light to highly confined plasmonic gap modes via nanoantennas," Applied Physics Letters, vol. 98, no. 10, Article ID 101109, 3 pages, 2011.

[130] A. Andryieuski, R. Malureanu, G. Biagi, T. Holmgaard, and A. Lavrinenko, "Compact dipole nanoantenna coupler to plasmonic slot waveguide," Optics Letters, vol. 37, no. 6, pp. 1124-1126, 2012.

[131] A. Alù and N. Engheta, "Wireless at the nanoscale: optical interconnects using matched nanoantennas," Physical Review Letters, vol. 104, no. 21, Article ID 213902, 4 pages, 2010.

[132] Z. Xiao, F. Luan, T.-Y. Liow, J. Zhang, and P. Shum, "Design for broadband high-efficiency grating couplers," Optics Letters, vol. 37, no. 4, pp. 530-532, 2012.

[133] D. Vermeulen, S. Selvaraja, P. Verheyen et al., "Highefficiency fiber-to-chip grating couplers realized using an advanced CMOS-compatible Silicon-on-Insulator platform," Optics Express, vol. 18, no. 17, pp. 18278-18283, 2010.

[134] L. Zhu, V. Karagodsky, and C. Chang-Hasnain, "Novel high efficiency vertical to in-plane optical coupler," in High Contrast Metastructures, vol. 8270 of Proceedings of SPIE, San Francisco, Calif, USA, 2012.

[135] Z. Cheng, X. Chen, C. Y. Wong et al., "Focusing subwavelength grating coupler for mid-infrared suspended membrane waveguide," Optics Letters, vol. 37, no. 7, pp. 1217-1219, 2012.

[136] J. Andkjær, S. Nishiwaki, T. Nomura, and O. Sigmund, "Topology optimization of grating couplers for the efficient excitation of surface plasmons," Journal of the Optical Society of America B, vol. 27, no. 9, pp. 1828-1832, 2010.
[137] M. W. Maqsood, R. Mehfuz, and K. J. Chau, "Highthroughput diffraction-assisted surface-plasmon-polariton coupling by a super-wavelength slit," Optics Express, vol. 18, no. 21, pp. 21669-21677, 2010.

[138] E. Verhagen, A. Polman, and L. Kuipers, "Nanofocusing in laterally tapered plasmonic waveguides," Optics Express, vol. 16, no. 1, pp. 45-57, 2008.

[139] X. Chen and H. K. Tsang, "Polarization-independent grating couplers for silicon-on-insulator nanophotonic waveguides," Optics Letters, vol. 36, no. 6, pp. 796-798, 2011.

[140] N. Talebi, M. Shahabadi, W. Khunsin, and R. Vogelgesang, "Plasmonic grating as a nonlinear converter-coupler," Optics Express, vol. 20, no. 2, pp. 1392-1405, 2012.

[141] I. M. Vellekoop, A. Lagendijk, and A. P. Mosk, "Exploiting disorder for perfect focusing," Nature Photonics, 2010.

[142] A. Alù and N. Engheta, "Input impedance, nanocircuit loading, and radiation tuning of optical nanoantennas," Physical Review Letters, vol. 101, no. 4, Article ID 043901, 4 pages, 2008.

[143] K. B. Crozier, A. Sundaramurthy, G. S. Kino, and C. F. Quate, "Optical antennas: resonators for local field enhancement," Journal of Applied Physics, vol. 94, no. 7, Article ID 4632, 11 pages, 2003.

[144] R. M. Bakker, A. Boltasseva, Z. Liu et al., "Near-field excitation of nanoantenna resonance," Optics Express, vol. 15, no. 21, pp. 13682-13688, 2007.

[145] L. Novotny, "Effective wavelength scaling for optical antennas," Physical Review Letters, vol. 98, no. 26, Article ID 266802, 4 pages, 2007.

[146] C. E. Talley, J. B. Jackson, C. Oubre et al., "Surface-enhanced Raman scattering from individual $\mathrm{Au}$ nanoparticles and nanoparticle dimer substrates," Nano Letters, vol. 5, no. 8, pp. 1569-1574, 2005.

[147] J. J. Greffet, "Nanoantennas for light emission," Science, vol. 308, no. 5728, pp. 1561-1563, 2005.

[148] M. Schnell, A. García-Etxarri, A. J. Huber, K. Crozier, J. Aizpurua, and R. Hillenbrand, "Controlling the nearfield oscillations of loaded plasmonic nanoantennas," Nature Photonics, vol. 3, no. 5, pp. 287-291, 2009.

[149] J. A. Schuller, E. S. Barnard, W. Cai, Y. C. Jun, J. S. White, and M. L. Brongersma, "Plasmonics for extreme light concentration and manipulation," Nature Materials, vol. 9, no. 3, pp. 193-204, 2010.

[150] L. Novotny and N. Van Hulst, "Antennas for light," Nature Photonics, vol. 5, no. 2, pp. 83-90, 2011.

[151] M. Klemm, "Novel directional nanoantennas for singleemitter sources and wireless nano-links," International Journal of Optics, vol. 2012, Article ID 348306, 7 pages, 2012.

[152] Q. H. Park, "Optical antennas and plasmonics," Contemporary Physics, vol. 50, no. 2, pp. 407-423, 2009.

[153] E. Cubukcu and F. Capasso, "Optical nanorod antennas as dispersive one-dimensional Fabry-Pérot resonators for surface plasmons," Applied Physics Letters, vol. 95, no. 20, Article ID 201101, 3 pages, 2009.

[154] P. Mühlschlegel, H. J. Eisler, O. J. F. Martin, B. Hecht, and D. W. Pohl, "Applied physics: resonant optical antennas," Science, vol. 308, no. 5728, pp. 1607-1609, 2005.

[155] P. Biagioni and B. Hecht, "Nanoantennas for visible and infrared radiation," Reports on Progress in Physics, vol. 57, no. 2, Article ID 024402, 2011.

[156] C. Balanis, Antenna Theory: Analysis and Design, WileyInterscience, 3th edition, 2005. 
[157] A. J. Ward and J. B. Pendry, "Refraction and geometry in Maxwell's equations," Journal of Modern Optics, vol. 43, no. 4, pp. 773-793, 1996.

[158] D. M. Shyroki, "Note on transformation to general curvilinear coordinates for Maxwell's curl equations (Is the magnetic field vector axial?)," http://arxiv.org/abs/physics/0307029v2.

[159] U. Leonhardt and T. G. Philbin, "Chapter 2 transformation optics and the geometry of light," Progress in Optics, vol. 53, pp. 69-152, 2009.

[160] J. B. Pendry, D. Schurig, and D. R. Smith, "Controlling electromagnetic fields," Science, vol. 312, no. 5781, pp. 17801782, 2006.

[161] J. Zhang, Y. Luo, and N. A. Mortensen, "Transmission of electromagnetic waves through sub-wavelength channels," Optics Express, vol. 18, no. 4, pp. 3864-3870, 2010.

[162] A. V. Kildishev and V. M. Shalaev, "Engineering space for light via transformation optics," Optics Letters, vol. 33, no. 1, pp. 43-45, 2008.

[163] E. E. Narimanov and A. V. Kildishev, "Optical black hole: broadband omnidirectional light absorber," Applied Physics Letters, vol. 95, no. 4, Article ID 041106, 3 pages, 2009.

[164] M. P. Bendsøe and O. Sigmund, Topology Optimization: Theory, Methods, and Applications, Springer, 2003.

[165] J. S. Jensen and O. Sigmund, "Topology optimization for nano-photonics," Laser and Photonics Reviews, vol. 5, no. 2, pp. 308-321, 2011.

[166] M. Pu, L. Yang, L. H. Frandsen et al., "Topology-optimized slow-light couplers for ring-shaped photonic crystal waveguide," in Proceedings of the Conference on Optical Fiber Communication, Collocated National Fiber Optic Engineers Conference (OFC/NFOEC '10), San Diego, Calif, USA, March 2010.

[167] R. Salgueiro and Y. S. Kivshar, "Nonlinear couplers with tapered plasmonic waveguides," Optics Express, vol. 20, no. 9, pp. 187-189, 2012. 

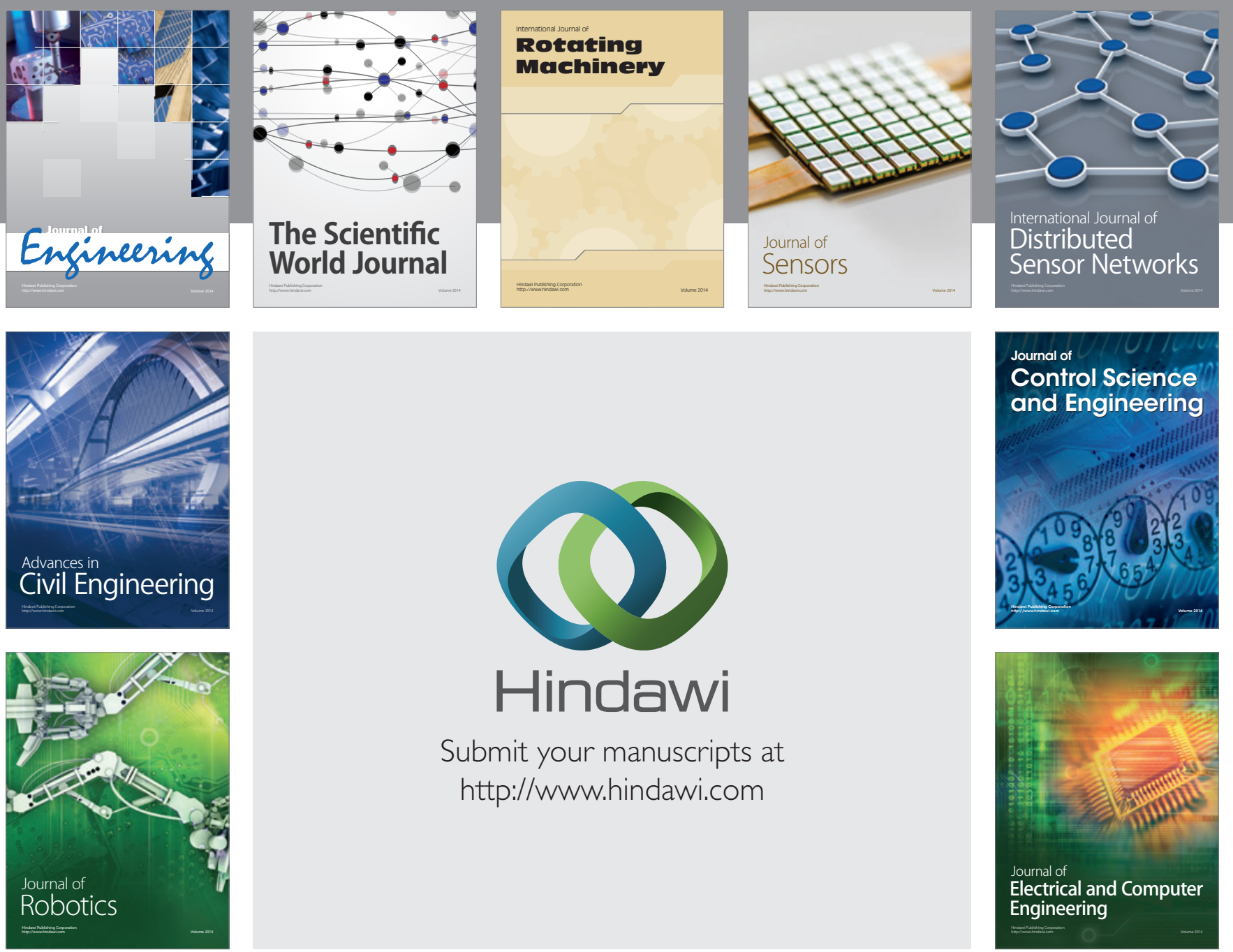

Submit your manuscripts at

http://www.hindawi.com
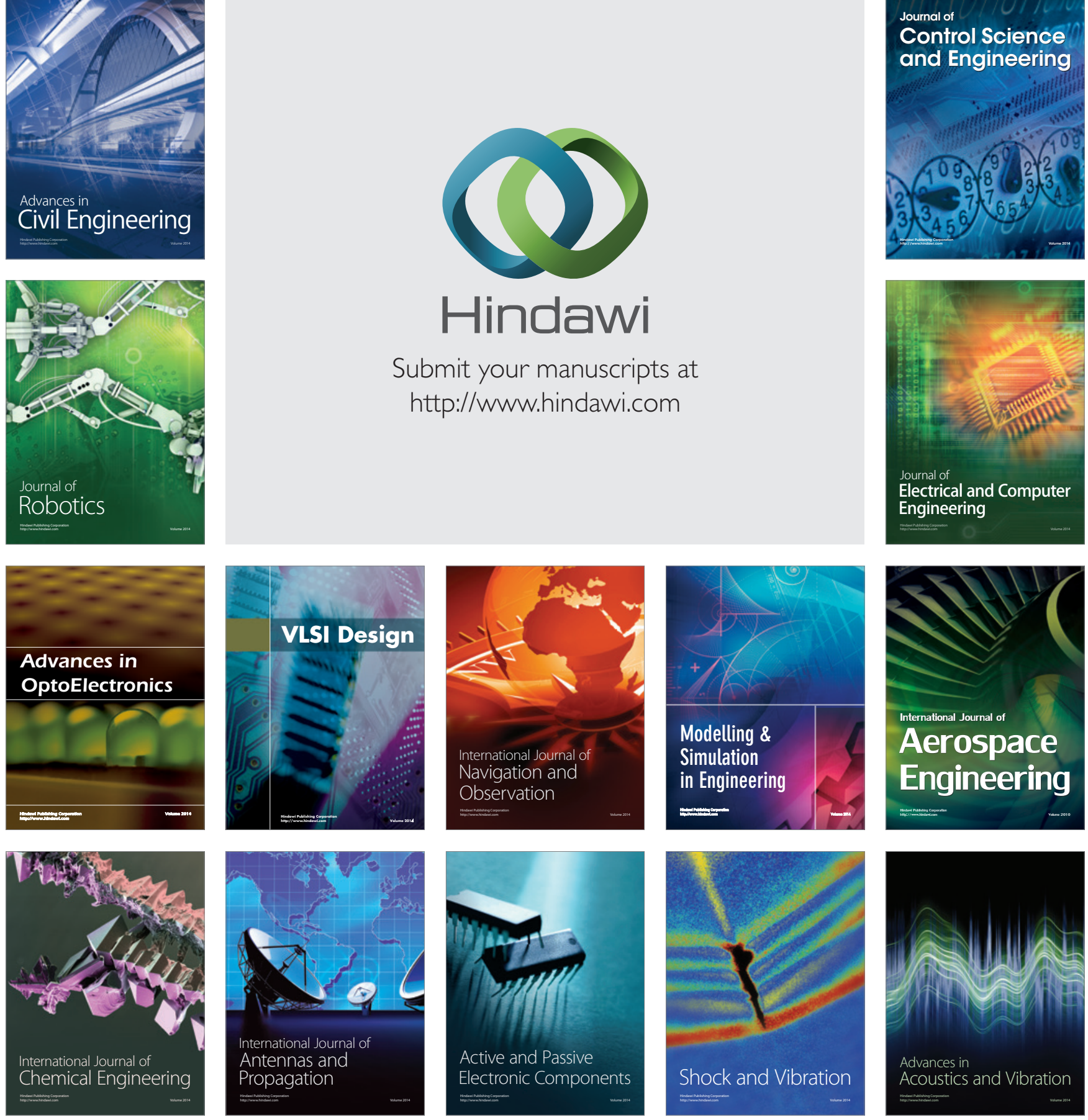